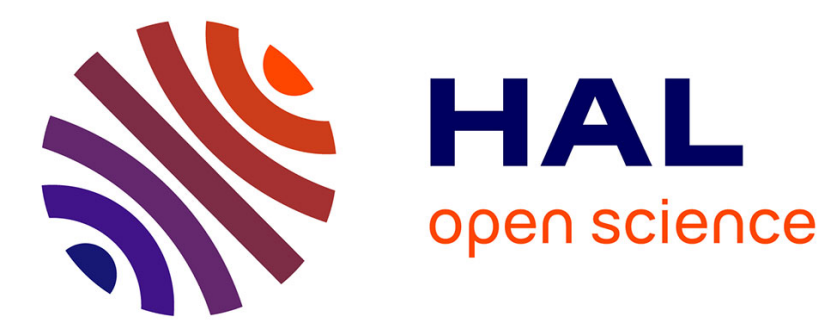

\title{
Grammars and clique-width bounds from split decompositions
}

\author{
Bruno Courcelle
}

\section{To cite this version:}

Bruno Courcelle. Grammars and clique-width bounds from split decompositions. Discrete Applied Mathematics, inPress, 10.1016/j.dam.2019.07.001 . hal-02093211v2

HAL Id: hal-02093211

https://hal.science/hal-02093211v2

Submitted on 31 Jul 2019

HAL is a multi-disciplinary open access archive for the deposit and dissemination of scientific research documents, whether they are published or not. The documents may come from teaching and research institutions in France or abroad, or from public or private research centers.
L'archive ouverte pluridisciplinaire HAL, est destinée au dépôt et à la diffusion de documents scientifiques de niveau recherche, publiés ou non, émanant des établissements d'enseignement et de recherche français ou étrangers, des laboratoires publics ou privés. 


\title{
Grammars and clique-width bounds from split decompositions
}

\author{
Bruno Courcelle \\ Labri, CNRS and Bordeaux University* \\ 33405 Talence, France \\ email: courcell@labri.fr
}

July 1, 2019

\begin{abstract}
Graph decompositions are important for algorithmic purposes and for graph structure theory. We relate the split decomposition introduced by Cunnigham to vertex substitution, graph grammars and clique-width.

For this purpose, we extend the usual notion of substitution, upon which modular decomposition is based, by considering graphs with dead (or non-boundary) vertices. We obtain a graph grammar for distancehereditary graphs consisting of four rules collected in a single equation. We also bound the clique-width of a graph in terms of those of the components of a split decomposition that need not be canonical.

For extending these results to directed graphs and their split decompositions (that we handle formally as graph-labelled trees), we need another extension of substitution : instead of two types of vertices, dead or alive as for undirected graphs, we need four types, in order to encode edge directions. We bound linearly the clique-width of a directed graph $G$ in terms of the maximal clique-width of a component arising in a graph-labelled tree that defines $G$. This result concerns all directed graphs, not only the strongly connected ones considered by Cunningham.
\end{abstract}

\section{Introduction}

Hierarchical graph decompositions and the associated graph complexity measures such as tree-width and clique-width are important for algorithmic purposes and graph structure theory. Tree-width and clique-width occur as parameters

*This work has been supported by the French National Research Agency (ANR) within the IdEx Bordeaux program "Investments for the future", CPU, ANR-10-IDEX-03-02, and also within the project GraphEn started in October 2015. 
in many fixed-parameter tractable (FPT) algorithms [14, 17, 20, 24], in particular for the verification of monadic second-order properties of graphs. They are actually linearly related in many interesting cases [10, 11, 26].

These algorithms are based on the construction of finite automata that run on algebraic terms representing tree-decompositions, or on clique-width terms in the case where the parameter is clique-width (see Definition 1.2). However, these automata cannot be implemented in the classical way because their sets of states are much to large. The notion of fly-automaton [12,13] can overcome this difficulty in many cases ${ }^{1}$ : these automata compute their transitions instead of looking into transition tables.

Even for checking properties of graphs of bounded tree-width, it is convenient to input graphs by clique-width terms, and we develop the theory and practice of fly-automata for graphs defined in this way in $[12,13]$. As for tree-width, computing the exact clique-width of a graph is an NP-complete problem [23]. However, clique-width terms witnessing "good" approximations of clique-width can be used with fly-automata. Such terms can be constructed by different algorithms, and with help of modular or split decomposition ${ }^{2}$, in preliminary steps. Let us point a difference between the two : modular decomposition is based on a rooted tree, and is clearly hierachical. Split decomposition is based on trees without root ; by choosing a root for such a tree, one can turn the decomposition into a hierachical one, but one needs an appropriate notion of graph substitution : we define one in this article. Rank-width, in a similar way, is based on unrooted trees. However, by choosing a root and using appropriate graph operations, derived from those upon which clique-width is defined, one obtains also a hierachical decomposition [16].

Modular decomposition is related to clique-width as follows. Each undirected graph has a canonical (i.e., unique up to isomorphism) modular decomposition. Its clique-width is the maximal clique-width of a prime module of the modular decomposition (Proposition 2.112 of [14]). It has also a canonical split decomposition. Theorem 4.14 establishes that its clique-width is linearly bounded in terms of the maximal clique-width of a prime component of this decomposition $^{3}$, the other components being stars and cliques. Theorem 5.14 shows the same for directed graphs. These theorems improve boundings based on logic or rank-width (cf. Sections 4.4 and 5.3).

Our initial motivating example was the class of distance-hereditary graphs ( $D H$ graphs in short). They are the undirected graphs $G$ in which the distance in any connected induced subgraph is the same as in $G$. They are known to have clique-width at most $3[29,36,37]$. However, recognizing the clique-width terms that define them is not easy. For the purpose of testing fly-automata, one may wish to generate large "random" DH graphs together with the algebraic terms of clique-width 3 that denote them. A good tool consists in using a context-

\footnotetext{
${ }^{1}$ A software is developped by Irène Durand [21]. Some parts of it are accessible online [22].

${ }^{2}$ See [31] and the references in that article for modular decomposition. Split decomposition defined by Cunnigham [18] is studied in [7, 27,28]. We give definitions in Sections 4 and 5 . Modular and split decomposition can both be computed in linear time for undirected graphs.

${ }^{3}$ Prime components cannot be split, cf. Section 4.1 .
} 
free graph grammar, built from clique-width operations that use three labels ${ }^{4}$. The characterization of DH graphs from [1], based on the addition of pendant edges and twins (see Definition 1.1), uses rewriting rules that are not those of a context-free graph grammar appropriate for using fly-automata intended to run on their derivation trees or on the equivalent clique-width terms. However, from this characterization, we can construct such a context-free grammar based on vertex-replacement, a notion developped in [14] and in [6], where an axiomatic definition of context-free graph grammars is given.

This construction uses a generalization of the standard notion of substitution of a graph for a vertex, that underlies the theory of modular decomposition. We distinguish in a graph $H$ some vertices as alive and the others as dead. Dead vertices will not be linked to any others in case $H$ is substituted into another graph ${ }^{5}$. We use this notion of substitution to define an associated notion of context-free vertex-replacement grammar (see $[6,14]$ for these graph grammars defined in a more general setting). We obtain a very simple grammar for DH graphs. Distance-hereditary graphs are also easily characterized in terms of their canonical split decompositions, and our grammar is based on these decompositions.

Generalizing this observation, we use grammars to generate the graphs whose components relative to split decomposition belong (up to isomorphism) to a fixed finite set $\mathcal{M}$. We bound their clique-width in terms of the maximal clique-width of a graph in $\mathcal{M}$, in a better way than what is known from [7].

Split decomposition ${ }^{6}$ for strongly connected (directed) graphs has also been studied in $[7,18]$. We extend to directed graphs our results for undirected graphs. For expressing split decompositions in terms of graph substitution, we need a more involved notion of substitution. Whereas for undirected graphs, we distinguish dead vertices from live ones (the others), for directed graphs, we need three types of live vertices, in order to encode three types of connections between two vertices $u$ and $v$ : from $u$ to $v$ (only), from $v$ to $u$ (only) or in both directions.

We consider split decompositions of directed graphs that need not be strongly connected, and we handle them formally as graph-labelled trees, a notion used in $[7,27]$. We prove that the clique-width of a directed graph $G$ is bounded by $8 k+1$, where $k$ is the maximum clique-width of a component of a graph-labelled tree that defines $G$.

Some properties of undirected graphs do not extend immediately to directed ones. Each component of a graph-labelled tree that defines an undirected graph $G$ is isomorphic to an induced subgraphs of $G$, hence has no larger clique-width.

\footnotetext{
${ }^{4}$ But we do not get an equal probability for two DH graphs of same size. See Section 4.5 for an unambigous grammar.

${ }^{5}$ In [36], dead vertices are defined in clique-width terms by inactive labels. We will use $\perp$ as an inactive label. Dead vertices may be called non-boundary by analogy with the case of graphs of bounded tree-width, built by gluing small graphs at boundary vertices [20], also called sources in [14].

${ }^{6} \mathrm{~A}$ different notion of split decomposition for directed graphs, that generalizes also the one for undirected graphs, has been defined in [34]. We will say a few words about it in Section 5.4 .
} 
For strongly connected directed graphs, each such component $H$ is isomorphic to a minor of the considered graph $G$. We obtain a bounding of the form ${ }^{7}$ $\operatorname{cwd}(H) \leq f(\operatorname{cwd}(G))$ where $f$ is a fixed exponential function. Improving this bound is an open problem.

To summarize, the purpose of this article is to clarify the close relationships between split decomposition, clique-width and vertex-replacement graph grammars based on vertex substitutions. In particular, we translate split decompositions of undirected graphs into graph grammars and we bound linearly the clique-width of a decomposed graph, either directed or not, in terms of those of the components. Our graph grammars can be used for counting graphs of special types and for random generation, along the lines of, e.g., [4, 25, 38].

Section 1 is devoted to basic definitions. In particular, we present our view of context-free graph grammars in terms of equation systems by using the example of cographs. Section 2 introduces vertex substitutions for undirected graphs with dead vertices, and the corresponding grammars. Section 3 relates clique-width and substitutions. Section 4 studies split decomposition of undirected graphs in this perspective, with the help of graph-labelled trees. Section 5 develops the case of directed graphs.

Acknowledgement: I thank M. Bousquet-Mélou, I. Durand, E. Gioan, M. Kanté, S. Oum, C. Paul and the referees for their useful comments and suggestions (in particular the reference to [4]). I also thank the editors of the special issue of Discrete Applied Mathematics relative to the GROW meeting in Toronto, in 2017 for wellcoming this submission, and the Fields Institute for supporting this excellent workshop.

\section{Graphs and clique-width}

Most definitions are well-known, we mainly review notation. We state a few facts that are either well-known or easy to prove.

The union of two sets is denoted by $\uplus$ in cases where we stress they are disjoint. The cardinality of a set $X$ is denoted by $|X|$ and its powerset by $\mathcal{P}(X)$. The set of integers $\{1, \ldots, n\}$ is denoted by $[n]$.

All trees and graphs are finite.

Trees

The set of nodes of a tree $T$ is denoted by $N_{T}$, and its set of leaves, i.e., of nodes of degree 1 , by $L_{T}$. A node that is not a leaf is internal.

If $T$ has a root, then $<_{T}$ denotes the corresponding ancestor relation, a strict partial order on $N_{T}$ (a node is not an ancestor of itself). The root, denoted by $\operatorname{root}_{T}$, is the unique maximal element and the leaves different from the root are

${ }^{7}$ Although in general, edge contraction does not preserve bounded clique-width , [9]. 
the minimal ones. A star $S_{n}$ is a tree with $n-1$ leaves linked to a single node called its center, where $n \geq 3$.

\section{Graphs}

We consider finite simple graphs, i.e., that are loop-free and without parallel edges. Graphs ${ }^{8}$ are directed or not. Directed edges are called arcs. A graph $G$ has a vertex set $V_{G}$ included in a fixed countably infinite set $\mathcal{V}$. Its set of edges or arcs is denoted by $E_{G}$. The corresponding binary adjacency relation is denoted by $e d g_{G}$ (even if $G$ is directed). If $G$ is undirected, we denote by $u v$, equvalently by $v u$, an edge linking vertices $u$ and $v$. If $G$ is directed, we denote by $u v$ an arc from $u$ to $v$. We also write $u-v$ or $u \rightarrow v$ to indicate that $u v$ is, respectively, an edge or an arc.

We denote by $H \subseteq_{i} G$ that $H$ is an induced subgraph of $G$, by $G[X]$ the induced subgraph of $G$ with vertex set $X \subseteq V_{G}$, by $G-X$ the graph $G\left[V_{G}-X\right]$ and by $G-x$ the graph $G\left[V_{G}-\{x\}\right]$ where $x \in V_{G}$.

Definition 1.1 : Distance-hereditary graph

An undirected graph $G$ is distance-hereditary (DH in short) if the distance of two vertices in every connected induced subgraph is the same as in $G$. For an example, the cycle $C_{4}$ (with 4 vertices) is $\mathrm{DH}$ whereas $C_{5}$ is not. The $\mathrm{DH}$ graphs are characterized as follows ${ }^{9}:$ a DH graph is an isolated vertex, or the disjoint union of two DH graphs or is obtained from a DH graph by the addition of a pendant edge to a vertex $x$, or of a true or false twin to $x$. Adding to $x$ a true twin is adding a new vertex $y$ linked to $x$ and to the neighbours of $x$. Adding a false twin is similar with $y$ not linked to $x$.

\section{Clique-width}

Clique-width is based on operations that modify or combine vertex-labelled graphs. There will be some restrictions regarding the special label $\perp$ to be used in Section 3.

Definition 1.2 : Labelled graphs and clique-width

(a) Let $C$ be a finite set of labels. A $C$-labelled graph, or simply, a $C$-graph, is a triple $G=\left(V_{G}, E_{G}, \pi_{G}\right)$ where $\pi_{G}$ is a mapping : $V_{G} \rightarrow C$. Its type, denoted by $\tau(G)$, is $\pi_{G}\left(V_{G}\right)$, i.e., the finite set of labels from $C$ that label some vertex of $G$.

We denote by $\simeq$ the isomorphism of $C$-graphs up to vertex labels, i.e., the isomorphism of the underlying unlabelled graphs, and by $\equiv$ the existence of an isomorphism that respects labels. An abstract C-graph (resp. abstract graph) is an equivalence class of $\equiv$ (resp. of $\simeq$ ).

(b) We define operations on $C$-graphs :

\footnotetext{
${ }^{8}$ Undefined notions are as in [19].

${ }^{9}$ This characterization is from [1]. The DH graphs are also the graphs of rank-width 1 [37]. They have clique-width at most 3 , as we will prove in detail.
} 
- the union of two disjoint $C$-graphs ; it is denoted by the binary infix function symbol ${ }^{10} \oplus$,

- the unary operation $a d d_{a, b}$ for $a, b \in C, a \neq b$ adds an undirected edge between each $a$-labelled vertex $x$ and each $b$-labelled vertex $y$ (unless there is already an edge $x y$ ),

- for building directed graphs, we use similarly $\overrightarrow{a d d}_{a, b}$ to add arcs from $a$-labelled to $b$-labelled vertices,

- the unary operation ${ }^{11}$ relab $_{h}$ changes every vertex label $a$ into $h(a)$ where $h$ is a partial mapping : $C \rightarrow C$ (a label $a$ is not modified if $h(a)$ is undefined).

- for each $a \in C$, the nullary symbol $\mathbf{a}(x)$ denotes the isolated vertex $x(x \in \mathcal{V})$ labelled by $a$.

Hence, $\tau(G \oplus H)=\tau(G) \cup \tau(H), \tau\left(a d d_{a, b}(G)\right)=\tau\left(\overrightarrow{a d d}_{a, b}(G)\right)=\tau(G)$, $\tau\left(\operatorname{relab}_{h}(G)\right)=h^{\prime}(\tau(G))$ where $h^{\prime}$ is the total mapping such that $: h^{\prime}(a):=$ if $h(a)$ is defined then $h(a)$ else $a$. We have $\tau(\mathbf{a}(x))=\{a\}$.

(c) We denote by $F_{C}$ the countable set of these operations. A term over $F_{C}$ is well-formed if no two occurrences of nullary symbols denote the same vertex; in particular, the graphs defined by the two arguments of $\oplus$ are disjoint. We denote by $T\left(F_{C}\right)$ the set of well-formed terms, that we will call the clique-width terms. Each such term $t$ denotes a $C$-graph $\boldsymbol{v a l}(t)$ whose vertices are those specified by the nullary symbols of $t$. Its width is the number of labels that occur in $t$.

Using a standard convention, we will denote in the same way a function symbol and the graph operation it defines. Hence, relab $b_{h}(t)$ is a term if $t$ is a term in $T\left(F_{C}\right)$, and relab $_{h}(G)$ denotes a $C$-graph if $G$ denotes a $C$-graph.

(d) The clique-width of a $C$-graph ${ }^{12} G$, denoted by $c w d(G)$, is the least width of a term $t$ such that $G \simeq \operatorname{val}(t)$. We denote by $c w d^{*}(G)$ the least width of a term $t$ such that $G \equiv \boldsymbol{v a l}(t)$. Hence, $c w d(G) \leq c w d^{*}(G)$. Clearly, $c w d(G)=c w d^{*}\left(G^{\prime}\right)$ where $G^{\prime}$ is obtained from $G$ by relabelling all its vertices in the same way.

(e) If $G$ and $H$ are not disjoint, we define $G \oplus H$ as the union of two disjoint isomorphic copies of $G$ and $H$. The resulting $C$-graph is well-defined up to isomorphism, hence as an abstract $C$-graph.

Here are some examples (cf. [14]). The clique-width of a tree is at most 3, that of the clique $K_{n}$ is 2 for $n \geq 2$. The undirected cycles $C_{3}, C_{4}$ have cliquewidth $2, C_{5}, C_{6}$ have clique-width 3 , and $C_{n}$ has clique-width 4 for $n \geq 7$. For directed cycles $\vec{C}_{n}$, we have $\operatorname{cwd}\left(\vec{C}_{3}\right)=3$ and $\operatorname{cwd}\left(\vec{C}_{n}\right)=4$ if $n \geq 4$.

\footnotetext{
${ }^{10} \mathrm{As} \oplus$ is associative, we will write $t=t_{1} \oplus t_{2} \oplus \ldots \oplus t_{n}$ instead of $t_{1} \oplus\left(t_{2} \oplus\left(\ldots \oplus t_{n}\right) \ldots\right)$ or any equivalent writing. It is also comutative.

${ }^{11}$ If $h$ modifies only one label, we call relab $b_{h}$ an elementary relabelling. By using only elementary relabellings, one obtains the same notion of clique-width ([14], Proposition 2.118).

${ }^{12}$ In [14], we denote $c w d^{*}$ by $c w d$.
} 
Lemma 1.3 : For every $C$-graph $G$, we have :

$$
\max \{|\tau(G)|, \operatorname{cwd}(G)\} \leq c w d^{*}(G) \leq|\tau(G)| \cdot \operatorname{cwd}(G) .
$$

Proof: The first inequality is clear from definitions. To prove the second one, we assume without loss of generality, that the type of $G$ is $[p]$. Let $H$ be $G$ with all vertices labelled in the same way. Let $C$ be the set of $k$ labels of a term $t$ that defines $H$. For each $a$ in $C$ and $i \in[p]$, we define a new label $(a, i)$ that will only label the vertices $x$ such that $\pi_{G}(x)=i$.

Consider in $t$ a nullary symbol $\boldsymbol{a}(x)$. If $\pi_{G}(x)=i$, we replace it by $(\boldsymbol{a}, \boldsymbol{i})(x)$.

Each relabelling relab $b_{h}$ is replaced by relab $h_{h^{\prime}}$ where $h^{\prime}$ maps $(a, i)$ to $(b, i)$ whenever $h$ maps $a$ to $b$. Similarly, we replace $a d d_{a, b}$ by the composition of the operations $a d d_{(a, i),(b, j)}$ for $i, j \in[p]$. We obtain in this way a term ${ }^{13} t^{\prime}$ over the set of labels $[p] \uplus(C \times[p])$. We let $h^{\prime \prime}: C \times[p] \rightarrow[p]$ map $(a, i)$ to $i$ for $a \in C$ and $i \in[p]$. Then $G=\operatorname{relab}_{h^{\prime \prime}}\left(\boldsymbol{v a l}\left(t^{\prime}\right)\right)=\operatorname{val}\left(\operatorname{relab}_{h^{\prime \prime}}\left(t^{\prime}\right)\right)$. The term $\operatorname{relab}_{h^{\prime \prime}}\left(t^{\prime}\right)$ uses at most $p(1+k)$ labels. However, we can fix some $a \in C$ and replace everywhere $(a, i)$ by $i$, for each $i$. We obtain a term of width at most $p k$ that defines $G$.

Hence if the type of $G$ consists of $p$ labels and $k$ is the clique-width of the corresponding unlabelled graph, then one can define $G$, with its labelling, by a term with at most $p k$ labels. This lemma implies that $\operatorname{cwd}(G) \leq 2 c w d(G-x)$ if $x$ is a vertex of $G$. This bound is proved in [30].

Questions 1.4: Can one improve the bounds ${ }^{14} \operatorname{cwd}(G) \leq 2 c w d(G-x)$ and $|\tau(G)| \cdot \operatorname{cwd}(G)$ (of Lemma 1.3)?

Definition 1.5 : Abstract graphs

We will also use nullary "generic" symbols a that do not denote any particular vertex. The vertex defined by an occurrence ${ }^{15} u$ of $\mathbf{a}$ in a term $t$ is $u$ itself. We will also consider that a term written with such nullary symbols denotes an abstract $C$-graph (cf. Definition 1.2(a,e)). See [14], Section 2.52 .

We will denote by $\bar{F}_{C}$ the signature $F_{C}$ where each symbol $\mathbf{a}(x)$ is replaced by $\mathbf{a}$, and by $\bar{t}$ the term in $T\left(\bar{F}_{C}\right)$ obtained from a term $t \in T\left(F_{C}\right)$ by replacing each $\mathbf{a}(x)$ by $\mathbf{a}$. Then $\boldsymbol{v a l}(\bar{t}) \equiv \boldsymbol{v} \boldsymbol{a l}(t)$.

Example 1.6 : The grammar for cographs.

We present context-free graph grammars, defined as equation systems whose unknowns are sets of abstract graphs, by using the example of cographs.

(1) One characterization of cographs is the following recursive one. Graphs are simple and undirected. The join of two disjoint undirected graphs $G$ and

\footnotetext{
${ }^{13}$ The construction is similar for directed graphs and it needs no more labels.

${ }^{14}$ It not hard to prove that $l c w d(G) \leq \operatorname{lcwd}(G-x)+2$ where $l c w d$ denotes the linear cliquewidth. This variant is defined by requiring that at least one of the two arguments of $\oplus$ is a nullary symbol. See e.g., [14, 32].

${ }^{15}$ Occurrences in terms can be designated by Dewey words or by integers, cf. Definition 2.3 in $[14]$,
} 
$H$, denoted by $G \otimes H$, is defined as their union augmented with edges between any vertex of $G$ and any vertex of $H$.

A cograph is either an isolated vertex, $G \oplus H$ or $G \otimes H$ for disjoint cographs $G$ and $H$. Hence, the set $\mathcal{C}$ of abstract cographs is the least set (least for inclusion) that satisfies the recursive equation :

$$
\mathcal{C}=\{*\} \cup(\mathcal{C} \oplus \mathcal{C}) \cup(\mathcal{C} \otimes \mathcal{C})
$$

where $*$ denotes an isolated vertex (up to isomorphism), $\mathcal{D} \oplus \mathcal{E}:=\{G \oplus H \mid$ $G \in \mathcal{D}, H \in \mathcal{E}\}$ and similarly for $\otimes$.

We call such a description a (context-free) graph grammar.

Each cograph has thus a hierchical description, in terms of smaller cographs and the two operations $\oplus$ and $\otimes$. Hence, it is defined by, or more formally, is the value of a term in $T(\{\oplus, \otimes, *\})$, i.e., a term written with $\oplus, \otimes$ and $*$. For example the term $t:=((* \oplus *) \oplus *) \otimes(* \oplus *)$ defines the complete bipartite graph $K_{3,2}$.

A fundamental property ([14], Proposition 3.23) states that the same recursive equation in sets of terms $X \subseteq T(\{\oplus, \otimes, *\})$, hence :

$$
X=\{*\} \cup(X \oplus X) \cup(X \otimes X)
$$

defines (by taking the least solution) the terms representing cographs. (If $Y, Z \subseteq T(\{\oplus, \otimes, *\})$, then $Y \oplus Z$ denotes the set of terms $t \oplus t^{\prime}$ such that $t \in Y$ and $t^{\prime} \in Z$.)

Actually, this equation defines the full set $T(\{\oplus, \otimes, *\})$. Cographs are the graphs defined by all terms in $T(\{\oplus, \otimes, *\})$. A cograph can be defined by several different terms, hence, this grammar is ambigous, which makes difficult its use for counting. However, an unambigous grammar can be used as we will see in Section 4.5.

The general notion of a grammar allows systems of mutually recursive equations that define sets of graphs or sets of terms. An example is :

$$
\begin{aligned}
& \mathcal{D}=\{* \otimes *\} \cup(\mathcal{D} \oplus \mathcal{E}) \cup(\mathcal{E} \otimes \mathcal{E}), \\
& \mathcal{E}=\{*\} \cup(\mathcal{D} \oplus *) \cup(\mathcal{E} \otimes \mathcal{E}) .
\end{aligned}
$$

The least sets $\mathcal{D}$ and $\mathcal{E}$ satisfying these equations are particular sets of cographs. The two sets of terms that form the least solution in $T(\{\oplus, \otimes, *\})$ of the (identical) system :

$$
\begin{aligned}
& Y=\{* \otimes *\} \cup(Y \oplus Z) \cup(Z \otimes Z), \\
& Z=\{*\} \cup(Y \oplus *) \cup(Z \otimes Z) .
\end{aligned}
$$

define the sets $\mathcal{D}$ and $\mathcal{E}$.

To simplify notation, we will write $*$ instead of $\{*\}$ and $* \otimes *$ instead of $\{* \otimes *\}$ in such equations, and similarly for terms without unknowns. The same letters $X, Y, Z \ldots$ will be used for sets of terms and the sets of graphs they denote. 
(2) Systems of recursive set equations, written with set union and the extensions of functions to sets, make sense in any F-algebra $\mathbb{M}=\left(M,\left(f_{\mathbb{M}}\right)_{f \in F}\right)$ where $M$ is a set equipped with functions $f_{\mathbb{M}}$ indexed by a functional signature $F$. Take for example $F$ consisting of $a, b, f, g, h$ of respective arities $0,0,1,2,3$. Then a system of equations like :

$$
\begin{aligned}
& \mathcal{D}=a \cup f(b) \cup f(\mathcal{D}) \cup g(\mathcal{E}, \mathcal{E}), \\
& \mathcal{E}=b \cup h(\mathcal{D}, \mathcal{E}, \mathcal{E}) \cup g(\mathcal{E}, \mathcal{D}),
\end{aligned}
$$

where $\mathcal{D}, \mathcal{E} \subseteq M$ has a least solution. Its least solution in subsets of $T(a, b, f, g, h)$ consists of two sets whose sets of values in $\mathbb{M}$ are $\mathcal{D}$ and $\mathcal{E}$.

Such sets are the equational sets of the algebra $\mathbb{M}$. This notion is relative to the algebraic structure specified by the operations $\left(f_{\mathbb{M}}\right)_{f \in F}$, cf. [14], Chapter 3 .

\section{Substitution to vertices}

In this section, we consider undirected graphs. We will adapt the definitions to directed graphs in Section 5 .

Let $C$ be a set of labels containing $\perp$. The vertices of a graph $G$ labelled by $\perp$ will be said to be dead; they form the set $V_{G}^{\text {dead }}$. The others, said to be alive form the set $V_{G}^{\text {live }}$. The unary operation $\kappa$, read $k i l l$, relabels all vertices by $\perp$, hence makes them dead.

Definition 2.1 : Substitution.

Let $K$ be a $C$-graph and $x_{1}, \ldots, x_{p}$ be pairwise distinct vertices. Let $H_{1}, \ldots$, $H_{p}$ be pairwise disjoint $C$-graphs, that are disjoint ${ }^{16}, n \geq 3$, from $K$. We define a $C$-graph $G:=K\left[x_{1} \leftarrow H_{1}, \ldots, x_{p} \leftarrow H_{p}\right]$ as follows ${ }^{17}$ :

$$
\begin{aligned}
& V_{G}:=\left(V_{K}-\left\{x_{1}, \ldots, x_{p}\right\}\right) \uplus V_{H_{1}} \uplus \ldots \uplus V_{H_{p}}, \\
& \pi_{G}(v):=\pi_{K}(v) \text { if } v \in V_{K}-\left\{x_{1}, \ldots, x_{p}\right\}, \\
& \pi_{G}(v):=\pi_{K}\left(x_{i}\right) \text { if } v \in V_{H_{i}}^{\text {live }}, \\
& \pi_{G}(v):=\perp \text { if } v \in V_{H_{i}}^{\text {dead }} .
\end{aligned}
$$

Its edges are as follows, for $u, v$ in $V_{G}$ :

$u v \in E_{G}$ if and only if :

either $u v \in E_{K}$ and neither $u$ nor $v$ is in $\left\{x_{1}, \ldots, x_{p}\right\}$,

or $u v \in E_{H_{i}}$ for some $i$,

or $u \in V_{K}, u x_{i} \in E_{K}$ and $v \in V_{H_{i}}^{\text {live }}$ (or vice-versa by exchanging $u$ and $v$ since we define undirected graphs, so that $u v$ and $v u$ designate the same edge),

or $u \in V_{H_{i}}^{\text {live }}, v \in V_{H_{j}}^{\text {live }}$ and $x_{i} x_{j} \in E_{K}($ so that $i \neq j)$.

\footnotetext{
${ }^{16}$ It is actually enough to assume that $\left(V_{K}-\left\{x_{1}, \ldots, x_{p}\right\}\right) \cap\left(V_{H_{1}} \uplus \ldots \uplus V_{H_{p}}\right)=\emptyset$.

${ }^{17} \mathrm{Read} " H_{i}$ is substituted to $x_{i}$ in $K "$ ".
} 
The type of $G$ is thus that of $K$, possibly augmented with $\perp$ if some $H_{i}$ has dead vertices : these vertices are dead in $G$. The labels of $K$ have no influence on the definition of the edges of $G$, they only specify, together with the labels of the graphs $H_{i}$, those of the resulting graph $G$. The labels of the $H_{i}$ 's other than $\perp$ do not contribute to the labelling of $G$ : if for each $i$, a mapping $h_{i}: C \rightarrow C$ satisfies $h_{i}(a)=\perp$ if and only if $a=\perp$, then :

$$
K\left[x_{1} \leftarrow H_{1}, \ldots, x_{p} \leftarrow H_{p}\right]=K\left[x_{1} \leftarrow \operatorname{relab}_{h_{1}}\left(H_{1}\right), \ldots, x_{p} \leftarrow \operatorname{relab}_{h_{p}}\left(H_{p}\right)\right] .
$$

If all vertices of $H_{p}$ are dead, then

$$
\begin{aligned}
& K\left[x_{1} \leftarrow H_{1}, \ldots, x_{p} \leftarrow H_{p}\right]=\left(K-x_{p}\right)\left[x_{1} \leftarrow H_{1}, \ldots, x_{p-1} \leftarrow H_{p-1}\right] \\
& \oplus H_{p} .
\end{aligned}
$$

Because of dead vertices, this notion of substitution differs from the classical one, used in particular in the theory of modular decomposition (see the survey [31]). If $K, H_{1}, \ldots, H_{p}$ have no dead vertices, then $K\left[x_{1} \leftarrow H_{1}, \ldots, x_{p} \leftarrow H_{p}\right]$ is the usual substitution, as in [14], Section 2.5. $V_{K}$.

Proposition 2.2 : Let $K, H_{1}, H_{2}$ be pairwise disjoint $C$-graphs and $x_{1} \in$

(1) If $x_{2}$ is another vertex of $K$, then $K\left[x_{1} \leftarrow H_{1}\right]\left[x_{2} \leftarrow H_{2}\right]=K\left[x_{1} \leftarrow\right.$ $\left.H_{1}, x_{2} \leftarrow H_{2}\right]$.

(2) If $x_{2} \in V_{H_{1}}$, then $K\left[x_{1} \leftarrow H_{1}\right]\left[x_{2} \leftarrow H_{2}\right]=K\left[x_{1} \leftarrow H_{1}\left[x_{2} \leftarrow H_{2}\right]\right]$.

Proof : (1) Straightforward verification from the definitions.

(2) Let $G:=K\left[x_{1} \leftarrow H_{1}\right]\left[x_{2} \leftarrow H_{2}\right]$ and $G^{\prime}:=K\left[x_{1} \leftarrow H_{1}\left[x_{2} \leftarrow H_{2}\right]\right]$.Clearly, $V_{G}=V_{G^{\prime}}$.

(2.1) We now compare edges. Let $u, v$ belong to $V_{G}$. If $u$ and $v$ are both, either in $V_{K}$, or in $V_{H_{1}}$ or in $V_{H_{2}}$, then $u v \in E_{G}$ if and only if $u v \in E_{G^{\prime}}$. Otherwise we distinguish three cases.

(i) $u \in V_{K}$ and $v \in V_{H_{1}}$; then, $u v \in E_{G}$ if and only if $u x_{1} \in E_{K}$ and $v$ is live in $H_{1}$, if and only if $u v \in E_{G^{\prime}}$.

(ii) $u \in V_{K}$ and $v \in V_{H_{2}}$; then, $u v \in E_{G}$ if and only if $u x_{2} \in E_{K\left[x_{1} \leftarrow H_{1}\right]}$ and $v$ is live in $H_{2}$. The condition $u x_{2} \in E_{K\left[x_{1} \leftarrow H_{1}\right]}$ is equivalent to : $u x_{1} \in E_{K}$ and $x_{2}$ is live in $H_{1}$.

Now $u v \in E_{G^{\prime}}$ if and only if $u x_{1} \in E_{K}$ and $v$ is live in $H_{1}\left[x_{2} \leftarrow H_{2}\right]$ which is true if and only if $v$ is live in $H_{2}$ and $x_{2}$ is live in $H_{1}$. Hence, $u v \in E_{G}$ if and only if $u v \in E_{G^{\prime}}$.

(iii) $u \in V_{H_{1}}-\left\{x_{2}\right\}$ and $v \in V_{H_{2}}$; then $u v \in E_{G}$ if and only if $u x_{2} \in E_{H_{1}}$ and $v$ is live in $H_{2}$, if and only if $u v \in E_{H_{1}\left[x_{2} \leftarrow H_{2}\right]}$, if and only if $u v \in E_{G^{\prime}}$.

Hence, $G$ and $G^{\prime}$ have the same edges.

(2.2) It remains to verify that $\pi_{G}=\pi_{G^{\prime}}$.

If $u \in\left(V_{K}-\left\{x_{1}\right\}\right) \uplus\left(V_{H_{1}}-\left\{x_{2}\right\}\right)$, then $\pi_{G}(u)=\pi_{G^{\prime}}(u)$ because $u$ is not affected by the substitutions to $x_{2}$.

If $u \in V_{H_{2}}$, then $\pi_{G}(u)=\perp$ if $u$ is dead in $H_{2}$; it is $\pi_{K\left[x_{1} \leftarrow H_{1}\right]}\left(x_{2}\right)$ otherwise. We have $\pi_{K\left[x_{1} \leftarrow H_{1}\right]}\left(x_{2}\right)=\perp$ if $x_{2}$ is dead in $H_{1}$, and otherwise, it is $\pi_{K}\left(x_{1}\right)$. 
Now, $\pi_{G^{\prime}}(u)=\perp$ if $u$ is dead in $H_{1}\left[x_{2} \leftarrow H_{2}\right]$ and it is $\pi_{K}\left(x_{1}\right)$ otherwise; observe that $u$ is dead in $H_{1}\left[x_{2} \leftarrow H_{2}\right]$ if and only if it is dead in $H_{2}$ or $x_{2}$ is dead in $H_{1}$. We obtain $\pi_{G}(u)=\pi_{G^{\prime}}(u)$ in this case; this value is either $\pi_{K}\left(x_{1}\right)$ or $\perp$ (if $u$ is dead in $H_{2}$ or $x_{2}$ is dead in $H_{1}$ ).

This completes the proof.

Properties (1) and (2) are called respectively commutativity and associativity of substitution in [6]. They are axioms for the definition of context-free graph grammars based on an abstract notion of substitution and on equation systems, as explained in Example 1.6. These grammars are particular vertex replacement grammars [14].

Definition 2.3 : Graph operations based on substitution.

(a) For each $C$-graph $K$ with vertex set enumerated as $\left\{x_{1}, \ldots, x_{p}\right\}$, we define as follows a $p$-ary graph operation ${ }^{18}$ on $C$-graphs denoted by $\sigma\left[K, x_{1}, \ldots, x_{p}\right]$ :

$$
\sigma\left[K, x_{1}, \ldots, x_{p}\right]\left(H_{1}, \ldots, H_{p}\right):=K\left[x_{1} \leftarrow H_{1}, \ldots, x_{p} \leftarrow H_{p}\right]
$$

where $H_{1}, \ldots, H_{p}$ are pairwise disjoint $C$-graphs that are disjoint from $K$. Note that the vertex set of $\sigma\left[K, x_{1}, \ldots, x_{p}\right]\left(H_{1}, \ldots, H_{p}\right)$ is $V_{H_{1}} \uplus \ldots \uplus V_{H_{p}}$.

If $H_{1}, \ldots, H_{p}$ are not pairwise disjoint, we replace them by isomorphic copies in a standard way (cf. Definitions $1.2(\mathrm{e})$ and 1.5, and Chapter 2 of [14]), so that $\sigma\left[K, x_{1}, \ldots, x_{p}\right]$ becomes a $p$-ary operation on abstract $C$-graphs.

(b) We denote by $\Sigma_{C}$ the countable set of these operations together with the nullary symbols $\boldsymbol{a}(x)$ (this symbol denote the $a$-labelled vertex $x \in \mathcal{V}$, as in Definition 1.2(b)). A term $t \in T\left(\Sigma_{C}\right)$ is well-formed if each vertex $x$ occurs at most once in some symbol $\boldsymbol{a}(x)$. It defines a $C$-graph $\boldsymbol{v} \boldsymbol{a l}(t)$ where (1) is used to evaluate $\sigma\left[K, x_{1}, \ldots, x_{p}\right]\left(H_{1}, \ldots, H_{p}\right)$.

(c) The signature $\bar{\Sigma}_{C}$ is obtained from $\Sigma_{C}$ by replacing, for each $a$, each symbol $\boldsymbol{a}(x)$ by $\boldsymbol{a}$. As for clique-width terms in $T\left(\bar{F}_{C}\right)$, each term in $T\left(\bar{\Sigma}_{C}\right)$ denotes an abstract $C$-graph.

We denote by relab $b_{a}$ the relabelling relab $b_{h}$ such that $h(\perp):=\perp$ and $h(b):=a$ if $b \neq \perp$.

Proposition 2.4 : Let $t, t^{\prime} \in T\left(\Sigma_{C}\right)$ and $x$ be a vertex in the $C$-graph $\boldsymbol{v a l}(t)$ defined in $t$ by $\boldsymbol{a}(x)$. Let $t^{\prime}$ be a term such that $V_{\boldsymbol{v a l}\left(t^{\prime}\right)} \cap\left(V_{\boldsymbol{v a l}(t)}-\{x\}\right)=\emptyset$. We have $: \operatorname{val}(t)\left[x \leftarrow \boldsymbol{v} \boldsymbol{a l}\left(t^{\prime}\right)\right]=\boldsymbol{v} \boldsymbol{a l}\left(t\left[\operatorname{relab}_{a}\left(t^{\prime}\right) / \boldsymbol{a}(x)\right]\right)$.

The $C$-graph $\boldsymbol{v} \boldsymbol{a l}(t)\left[x \leftarrow \boldsymbol{v} \boldsymbol{a l}\left(t^{\prime}\right)\right]$ is obtained by substituting in $\boldsymbol{v a l}(t)$ the $C$-graph $\boldsymbol{v a l}\left(t^{\prime}\right)$ to the vertex $x$. The term $t\left[\operatorname{relab}_{a}\left(t^{\prime}\right) / \boldsymbol{a}(x)\right]$ is obtained by substituting in $t$ the term relab $b_{a}\left(t^{\prime}\right)$ to the unique occurrence of $\boldsymbol{a}(x)$. It is welldefined because $V_{\boldsymbol{v a l}\left(t^{\prime}\right)} \cap\left(V_{\boldsymbol{v a l}(t)}-\{x\}\right)=\emptyset$ (cf. the first footnote in Definition $2.1)$.

\footnotetext{
${ }^{18}$ We use the same notation $\sigma\left[K, v_{1}, \ldots, v_{p}\right]$ for the $p$-ary function symbol and the corresponding operation. Cf. Definition 1.2(c).
} 
Proof : By induction on the structure of $t$.

If $t=\boldsymbol{a}(x)$, then $\boldsymbol{v a l}(t)\left[x \longleftarrow \boldsymbol{v a l}\left(t^{\prime}\right)\right]=\boldsymbol{a}(x)\left[x \longleftarrow \boldsymbol{v} \boldsymbol{v a l}\left(t^{\prime}\right)\right]=\operatorname{relab}_{a}\left(\boldsymbol{v a l}\left(t^{\prime}\right)\right)$

$=\boldsymbol{v a l}\left(\operatorname{relab}_{a}\left(t^{\prime}\right)\right)=\boldsymbol{v} \boldsymbol{v a l}\left(t\left[\operatorname{relab}_{a}\left(t^{\prime}\right) / \boldsymbol{a}(x)\right]\right)$.

Let now $t=\sigma\left[K, v_{1}, \ldots, v_{p}\right]\left(t_{1}, \ldots, t_{p}\right)$. Without loss of generality and to simplify notation, we assume that $\boldsymbol{a}(x)$ occurs in $t_{1}$. Then, for every term $s$ :

$$
\begin{aligned}
& t[s / \boldsymbol{a}(x)]=\sigma\left[K, v_{1}, \ldots, v_{p}\right]\left(t_{1}[s / \boldsymbol{a}(x)], t_{2}, \ldots, t_{p}\right) \text { and so } \\
& \boldsymbol{v} \boldsymbol{a l}(t[s / \boldsymbol{a}(x)])= \\
& K\left[v_{1} \leftarrow \boldsymbol{v} \boldsymbol{a l}\left(t_{1}[s / \boldsymbol{a}(x)]\right), v_{2} \leftarrow \boldsymbol{v} \boldsymbol{a l}\left(t_{2}\right), \ldots, v_{p} \leftarrow \boldsymbol{v} \boldsymbol{a l}\left(t_{p}\right)\right] .
\end{aligned}
$$

By induction :

$$
\boldsymbol{v a l}\left(t_{1}\left[\operatorname{relab}_{a}\left(t^{\prime}\right) / \boldsymbol{a}(x)\right]\right)=\boldsymbol{v} \boldsymbol{a l}\left(t_{1}\right)\left[x \leftarrow \boldsymbol{v a l}\left(t^{\prime}\right)\right],
$$

hence, Equality (2) where $s=\operatorname{relab}_{a}\left(t^{\prime}\right)$ yields

$$
\begin{aligned}
& \operatorname{val}\left(t\left[r e l a b_{a}\left(t^{\prime}\right) / \boldsymbol{a}(x)\right]\right)=K\left[v_{1} \leftarrow \boldsymbol{v a l}\left(t_{1}\right)\left[x \leftarrow \operatorname{val}\left(t^{\prime}\right)\right], \ldots, v_{p} \leftarrow\right. \\
& \left.\operatorname{val}\left(t_{p}\right)\right] \\
& =K\left[v_{1} \leftarrow \boldsymbol{v a l}\left(t_{1}\right), \ldots, v_{p} \leftarrow \boldsymbol{v a l}\left(t_{p}\right)\right]\left[x \leftarrow \boldsymbol{v a l}\left(t^{\prime}\right)\right] \text { by Propoposition } \\
& 2.2(2), \\
& =\operatorname{val}(t)\left[x \leftarrow \boldsymbol{v a l}\left(t^{\prime}\right)\right] .
\end{aligned}
$$

By using these operations, one can define graph grammars, formalized by systems of recursive equations in sets of abstract $C$-graphs, of which one takes least solutions (cf. Example 1.6 and [14], Chapters 3 and 4).

Definitions 2.5 : Some useful operations.

Here are some operations on $D$-graphs, where $D:=\{\perp, \top\}$ and $\top$ labels the live vertices. The first two equalities are mere observations.

$\kappa(H)=\sigma\left[K, x_{1}\right](H)$ where $K$ consists of the dead vertex $x_{1}$.

$H_{1} \oplus H_{2}=\sigma\left[K, x_{1}, x_{2}\right]\left(H_{1}, H_{2}\right)$ where $K$ consists of two isolated live vertices $x_{1}$ and $x_{2}$.

We define :

$H_{1} \otimes H_{2}:=\sigma\left[K, x_{1}, x_{2}\right]\left(H_{1}, H_{2}\right)$ where $K$ is the edge $x_{1} x_{2}$, and $x_{1}$ and $x_{2}$ are alive.

$\Lambda\left(H_{1}, H_{2}\right):=\sigma\left[K, x_{1}, x_{2}\right]\left(H_{1}, H_{2}\right)$ where $K$ is the edge $x_{1} x_{2}, x_{1}$ is alive and $x_{2}$ is dead.

The operations $\oplus$ and $\otimes$ are associative and commutative. Here are some other algebraic properties ${ }^{19}$ :

\footnotetext{
${ }^{19}$ We leave as an open problem to find a complete set of equational axioms for $\oplus, \otimes, \Lambda, \kappa, \top$.
} 


$$
\begin{aligned}
& \kappa(G \otimes H)=\kappa(\Lambda(G, H)), \\
& \kappa(G \oplus H)=\kappa(G) \oplus \kappa(H)=\Lambda(\kappa(G), H)=\Lambda(\kappa(G), \kappa(H)), \\
& \Lambda\left(G, H_{1} \oplus H_{2}\right)=\Lambda\left(\Lambda\left(G, H_{1}\right), H_{2}\right) .
\end{aligned}
$$

We let $\bar{\Sigma}_{d h}$ be the signature $\{\oplus, \otimes, \Lambda, \kappa, \top\} \subseteq \bar{\Sigma}_{D}$. For every vertex $x$, we have $\perp(x)=\kappa(T(x))$. Hence, we need not put the nullary symbol $\perp$ in $\bar{\Sigma}_{d h}$. Actually, a vertex introduced by $\perp(x)$ is dead from the very beginning and is isolated in the defined graph.

Examples 2.6 : Some grammars over $\bar{\Sigma}_{d h}$.

Grammars are defined as equation systems that define sets of abstract $D$ graphs.

(1) The following equation for cographs that we have already seen in Example 1.6, can be solved in sets of abstract $D$-graphs :

$$
X=\top \cup(X \oplus X) \cup(X \otimes X) .
$$

All vertices of the generated $D$-graphs are labelled by $\top$ because $\oplus$ and $\otimes$ do not introduce dead vertices. We recall that $X \oplus X:=\{G \oplus H \mid G, H \in X\}$ if $X$ is a set of labelled graphs and similarly for $\otimes$ and the other operations considered below.

As observed in Example 1.6, this equation can also be solved in $T(\{\oplus, \otimes, \top\})$. Its solution is a set of terms ${ }^{20}$ that we will denote by $L(X)$. More generally, for a system of set equations over a functional signature $F$ that has unknowns $X, Y, Z \ldots$, we will denote by $L(X), L(Y), L(Z) \ldots$ the associated sets of terms in $T(F)$. If the system is solved in an $F$-algebra $\mathbb{M}$, the corresponding sets of objects $X, Y, Z \ldots$ are the sets of values in $\mathbb{M}$ of the terms in $L(X), L(Y), L(Z) \ldots$.

(2) We turn a rooted tree (cf. Section 1) into a $\{\top, \perp\}$-graph such that the root is the only live node.

These trees are defined recursively as follows : a unique node $a$ is a tree with root $a$. If $A$ and $B$ are disjoint rooted trees with respective roots $a$ and $b$, than one obtains a rooted tree $C$ by taking the union of $A$ and $B$, linked by an edge $a b$, and $a$ is taken as root of $C$. Then, $C=\Lambda(A, B)$ if $A, B, C$ are as above. The equation that defines the set $R$ of rooted trees is thus :

$$
R=\top \cup \Lambda(R, R) .
$$

Another grammar for trees, consisting of two equations is :

$$
\begin{aligned}
& Y=\top \cup \Lambda(\top, Z), \\
& Z=Y \cup(Z \oplus Z) .
\end{aligned}
$$

\footnotetext{
${ }^{20}$ We call language a set of terms, whence the notation $L(X)$. A set of graphs is not called a language, in order to have a coherent terminology.
} 
Here, $Z$ defines the nonempty disjoint unions of rooted trees.

(3) The set $T$ of (unrooted) trees is defined by the equation $T=\kappa(R)$ or $T=\kappa(Y)$, with $R, Y, Z$ defined as in (2). For an example, the tree with nodes $u, v, w, x, y, z$, root $x$ and edges $x y, x z, x v, z u$ and $v w$ is defined by the term :

$$
\Lambda(\Lambda(\Lambda(\top(x), \top(y)), \Lambda(\top(z), \top(u))), \Lambda(\top(v), \top(w)))
$$

belonging to $L(R)$ or by the term :

$$
\Lambda(\top(x), \top(y) \oplus \Lambda(\top(z), \top(u)) \oplus \Lambda(\top(v), \top(w)))
$$

that belongs to $L(Y)$.

The paths with one live vertex at one end are defined by the equation

$$
P=\top \cup \Lambda(\top, P) .
$$

(4) We will prove in the next proposition that the equation

$$
W=\top \cup(W \oplus W) \cup(W \otimes W) \cup \Lambda(W, W)
$$
$1.1)$.

defines, up to vertex labels, the distance-hereditary graphs (cf. Definition

From these equations, we obtain that the rooted trees are defined by all terms in $T(\{\Lambda, \top\})$ or by certain terms in $T(\{\oplus, \Lambda, \top\})$, and that the distancehereditary graphs are defined by terms in $T(\{\oplus, \otimes, \Lambda, \top\})$. In these equations and the generated terms, the label $\perp$ for dead vertices does not appear explicily, but it is introduced by the operations $\Lambda$ and $\kappa$.

In the following description of distance-hereditary (DH) graphs, all vertices are defined as dead, equivalently, unlabelled. The following recursive definition of DH graphs has been established in [3], but we think interesting to prove it by using the concepts of the present article. We recall that equation systems always define abstract graphs.

Proposition 2.7 : (1) The distance-hereditary graphs form the set $X$ defined by the two equations :

$$
X=\kappa(W) \text { and } W=\top \cup(W \oplus W) \cup(W \otimes W) \cup \Lambda(W, W) .
$$

(2) The connected distance-hereditary graphs form the set $Y$ defined by the equation :

$$
Y=\kappa(\top) \cup \kappa(W \otimes W)
$$


and the equation of (1) that defines $W$.

Proof : (1) Note that ${ }^{21} L(W)=T(\{\oplus, \otimes, \Lambda, \top\})$. For both directions we will use the characterization of DH graphs recalled in Definition 1.1.

Claim 1 : Every DH graph $G$ is in the set $\operatorname{val}(X)=\operatorname{val}(\kappa(W))$.

Proof : We use induction on the number $n$ of vertices of $G$.

If $n=1$, then $G$ is a single dead vertex, hence $G \equiv \kappa(\top) \in \kappa(W)$.

Otherwise there are four cases.

(i) $G$ is the disjoint union of two DH graphs $H, H^{\prime}$. Then, $H \equiv \kappa(t), H^{\prime} \equiv$ $\kappa\left(t^{\prime}\right)$ for some $t, t^{\prime} \in W=T(\{\oplus, \otimes, \Lambda, \top\})$. Hence, $G \equiv \kappa\left(t \oplus t^{\prime}\right)$ where $t \oplus t^{\prime} \in$ $L(W)$ because $\kappa\left(t \oplus t^{\prime}\right) \equiv \kappa(t) \oplus \kappa\left(t^{\prime}\right)$.

(ii) If $G$ is obtained from a $\mathrm{DH}$ graph $G^{\prime}$ by adding a pendant vertex $y$ to a vertex $x$ of $G^{\prime}$, we have $G=G^{\prime}[x \leftarrow H]$ where $H$ is the edge $x y$, with $x$ alive and $y \operatorname{dead}^{22}$; hence $H=\boldsymbol{v a l}(\Lambda(\top(x), \top(y)))$.

We have $G^{\prime}=\kappa\left(\boldsymbol{v a l}\left(t^{\prime}\right)\right)$ where $t^{\prime}$ is a well-formed term over $\oplus, \otimes, \Lambda$ and the nullaries that define vertices. It has one occurrence of $T(x)$.

We let $t:=t^{\prime}[\Lambda(\top(x), \top(y)) / \top(x)]$. By Proposition 2.4, we have $\boldsymbol{v a l}(t)=$ $\boldsymbol{v a l}\left(t^{\prime}[\Lambda(\top(x), \top(y)) / \top(x)]\right)=\boldsymbol{v} \boldsymbol{v a l}\left(t^{\prime}\left[\operatorname{relab}_{\top}(\Lambda(\top(x), \top(y))) / \top(x)\right]\right)$

$\operatorname{val}\left(t^{\prime}\left[x \leftarrow \operatorname{val}\left(r e l a b_{\top}(\Lambda(\top(x), \top(y)))\right)\right]=\operatorname{val}\left(t^{\prime}[x \leftarrow H]\right)\right.$.

Hence $G=\kappa(t)$, so that $G \equiv \kappa(\bar{t})$ where $\bar{t} \in L(W)$ is obtained from $t$ by replacing by $\top$ the symbols $\top(z)$ that define vertices.

(iii) Let $G$ be obtained from a DH graph $G^{\prime}$ by adding a false twin $y$ to a vertex $x$. We have $G=G^{\prime}[x \leftarrow H]$ where $H$ consists of two isolated live vertices $x$ and $y$. Hence $H=\boldsymbol{v} \boldsymbol{a l}(\top(x) \oplus \top(y))$. The proof continues as in (ii) with $\top(x) \oplus \top(y)$ instead of $\Lambda(\top(x), \top(y))$.

(iv) Let $G$ be obtained from a DH graph $G^{\prime}$ by adding a true twin $y$ to a vertex $x$. Here $G=G^{\prime}[x \leftarrow H]$ where $H$ consists of two live vertices $x$ and $y$ linked by an edge, hence $H=\boldsymbol{v a l}(\top(x) \otimes \top(y))$. The proof continues as in (iii) with $\top(x) \otimes \top(y)$ instead of $\top(x) \oplus \top(y)$.

Claim 2 : If $G=\operatorname{val}(t)$ for some well-formed term $t$ over $\oplus, \otimes, \Lambda$ and the nullaries that define vertices, then $\kappa(G)$ is DH.

Proof : By induction on the size of $t$.

If $t=\mathrm{T}(x)$, the result holds because an isolated vertex is $\mathrm{DH}$. Otherwise, we can find a position $u$ in $t$ such that $t / u$, the subterm of $t$ issued from position $u$, is either $\Lambda(\top(x), \top(y)), \top(x) \oplus \top(y)$, or $\mathrm{\top}(x) \otimes \top(y)$. Then $t=t^{\prime}[(t / u) / \top(x)]$ for some well-formed term $t^{\prime}\left(t^{\prime}\right.$ is obtained by replacing in $t$ the subterm $t / u$ by $\mathrm{\top}(x))$. By induction, $\kappa\left(\boldsymbol{v} \boldsymbol{a l}\left(t^{\prime}\right)\right)$ is a DH graph $G^{\prime}$ and $G=G^{\prime}[x \leftarrow H]$ by Proposition 2.4, where $H$ is respectively as in cases (ii), (iii) or (iv)

(2) It is clear that a term $t$ in $L(X)$ defines a connected graph if and only if it is not of the form $\kappa\left(t_{1} \oplus t_{2}\right)$. Hence, the connected DH graphs can be defined by the equation :

\footnotetext{
${ }^{21}$ See Example 1.6 for the solution of equations in sets of terms. If $t$ is a term, we will write $G \equiv t$ to indicate that $G \equiv \boldsymbol{v a l}(t)$.

${ }^{22}$ We use here the footnote in Definition 2.1.
} 


$$
Y=\kappa(\top) \cup \kappa(W \otimes W) \cup \kappa(\Lambda(W, W))
$$

where $W$ is as in (1). However, we observed in Definition 2.5 that $\kappa(\Lambda(G, H))=$ $\kappa(G \otimes H)$ for all $D$-graphs $G$ and $H$. Hence, the term $\kappa(\Lambda(W, W))$ can be removed.

The bipartite $D H$ graphs are built from isolated vertices by the addition of pendant edges and of false twins [1]. Hence, they form the set $B$ defined by the two equations $B=\kappa\left(W^{\prime}\right)$ and $W^{\prime}=\top \cup\left(W^{\prime} \oplus W^{\prime}\right) \cup \Lambda\left(W^{\prime}, W^{\prime}\right)$.

\section{Clique-width and substitution operations.}

A derived operation $^{23}$ relative to an $F$-algebra $\mathbb{M}$ is defined by a term $t$ in $T\left(F,\left\{u_{1}, \ldots, u_{p}\right\}\right)$, i.e., a term over $F$ with variables (or indeterminates, i.e, nullary symbols to which values or terms can be substituted) $u_{1}, \ldots, u_{p}$. The corresponding $p$-ary function $t_{\mathbb{M}}$ is defined by evaluating $t$ with $p$ arguments from the domain of $\mathbb{M}$ as values of $u_{1}, \ldots, u_{p}$.

For an example using clique-width operations, the operation $\otimes$ on graphs of type $\{\top\}$ (cf. Definitions 1.6 and 2.5) satisfies the following equality for all $D$-graphs $G, H$ :

$$
G \otimes H=\operatorname{relab}_{a \rightarrow \top}\left(a d d_{\top, a}\left(G \oplus \operatorname{relab}_{\top \rightarrow a}(H)\right)\right) .
$$

Hence, $\otimes$ is a derived operation defined by the $\operatorname{term}^{24} \operatorname{relab}_{a \rightarrow \top}\left(a d d_{\perp, a}\left(u_{1} \oplus\right.\right.$ $\left.\left.r e l a b_{\top \rightarrow a}\left(u_{2}\right)\right)\right)$.

Our objective is to express the operations $\sigma\left[K, x_{1}, \ldots, x_{p}\right]$ as derived operations over $F_{C}$, the signature upon which clique-width is based.

We let $\operatorname{Lin}\left(F_{C},\left\{u_{q}, \ldots, u_{p}\right\}\right)$ be the set of terms in $T\left(F_{C},\left\{u_{q}, \ldots, u_{p}\right\}\right), q \leq p$, where each variable $u_{i}$ has a unique occurrence and no other nullary symbol occurs. Every such term defines a $(p-q+1)$-ary mapping on $C$-graphs denoted by $t_{\mathbb{G}}$. For pairwise disjoint graphs $H_{q}, \ldots, H_{p}$, the vertex set of $t_{\mathbb{G}}\left(H_{q}, \ldots, H_{p}\right)$ is $V_{H_{q}} \uplus \ldots \uplus V_{H_{p}}$.

We define $T_{\perp}\left(F_{C}\right)$ as the set of terms that use none of the operations ${ }^{25}$ $a d d_{a, \perp}, a d d_{\perp, a}, \overrightarrow{a d d}_{a, \perp}, \overrightarrow{a d d}_{\perp, a}$, relab $b_{h}$ if $h(\perp) \neq \perp$, and no nullary symbol $\perp(x)$. We denote by $c w d^{\perp}(G)$ the minimal cardinality of $C-\{\perp\}$ such that ${ }^{26}$ $G \equiv \boldsymbol{v a l}(t)$ for some term $t \in T_{\perp}\left(F_{C}\right)$. Clearly, $\operatorname{cwd}(G) \leq c w d^{\perp}(G)+1$. We have $c w d(T)=3$ and $c w d^{\perp}(T)=2$ for any tree $T$ that is not a star.

Let $K$ be a $C$-graph with vertex set $\left\{x_{q}, \ldots, x_{p}\right\}$ defined by a term $t$ in $T_{\perp}\left(F_{C}\right)$. Each vertex $x_{i}$ occurs in a nullary symbol $\boldsymbol{a}_{\boldsymbol{i}}\left(x_{i}\right)$ in $t$ such that $a_{\boldsymbol{i}} \neq \perp$. We define $\widehat{t}:=t\left[u_{q} / \boldsymbol{a}_{\boldsymbol{q}}\left(x_{q}\right), \ldots, u_{p} / \boldsymbol{a}_{\boldsymbol{p}}\left(x_{p}\right)\right] \in \operatorname{Lin}\left(F_{C},\left\{u_{q}, \ldots, u_{p}\right\}\right)$.

\footnotetext{
${ }^{23}$ See, e.g. [14], Section 2.1 .

${ }^{24}$ This term uses an auxiliary label $a \neq \perp, \top$. However, it defines graphs of type $\{\top\}$ from graphs of same type. The label $a$ can be replaced by any other label different from $\top$ or $\perp$.

${ }^{25}$ These limitations on the use of $\perp$ make it an inactive label in [36].

${ }^{26}$ The equivalence $\equiv$ respects vertex labels, cf. Definition 1.2(a).
} 
Lemma 3.1: Let $K$ be a $C$-graph with vertex set $\left\{x_{1}, \ldots, x_{p}\right\}$ defined by $t \in T_{\perp}\left(F_{C}\right)$. Let $\widehat{t}:=t\left[u_{1} / \boldsymbol{a}_{\mathbf{1}}\left(x_{1}\right), \ldots, u_{p} / \boldsymbol{a}_{\boldsymbol{p}}\left(x_{p}\right)\right]$. For pairwise disjoint $C$-graphs $H_{1}, \ldots, H_{p}$, we have:

$$
\sigma\left[K, x_{1}, \ldots, x_{p}\right]\left(H_{1}, \ldots, H_{p}\right)=\widehat{t}_{\mathbb{G}}\left(\operatorname{relab}_{a_{1}}\left(H_{1}\right), \ldots, \operatorname{relab}_{a_{p}}\left(H_{p}\right)\right) .
$$

Proof: By induction on the structure of $t$. We recall that $r e l a b_{a}$ is the relabelling that replaces by $a$ every label except $\perp$.

If $t=\boldsymbol{a}_{\mathbf{1}}\left(x_{1}\right)$, then $\widehat{t}=u_{1}, K$ consists of the $a_{1}$-vertex $x_{1}$ and $\sigma\left[K, x_{1}\right]\left(H_{1}\right)=$ $\operatorname{relab}_{a_{1}}\left(H_{1}\right)=\widehat{t}_{\mathbb{G}}\left(\operatorname{relab}_{a_{1}}\left(H_{1}\right)\right)$ (by the behaviour of labels in substitution).

If $t=t_{1} \oplus t_{2}$, then, without loss of generality, we assume that the vertices of $K_{1}:=\boldsymbol{v a l}\left(t_{1}\right)$ are $x_{1}, \ldots, x_{i}$ and those of $K_{2}:=\boldsymbol{v} \boldsymbol{v a l}\left(t_{2}\right)$ are $x_{i+1}, \ldots, x_{p}$. We have $\widehat{t}=\widehat{t_{1}} \oplus \widehat{t_{2}}$. Then, since substitution distributes over disjoint union ${ }^{27}$ and by induction :

$$
\begin{aligned}
& \sigma\left[K, x_{1}, \ldots, x_{p}\right]\left(H_{1}, \ldots, H_{p}\right)= \\
& \sigma\left[K_{1}, x_{1}, \ldots, x_{i}\right]\left(H_{1}, \ldots, H_{i}\right) \oplus \sigma\left[K_{2}, x_{i+1}, \ldots, x_{p}\right]\left(H_{i+1}, \ldots, H_{p}\right)= \\
& \widehat{t}_{\mathbb{G}}\left(\operatorname{relab}_{a_{1}}\left(H_{1}\right), \ldots, \operatorname{relab}_{a_{i}}\left(H_{i}\right)\right) \oplus \widehat{t_{2 \mathbb{G}}}\left(\operatorname{relab}_{a_{i}}\left(H_{i}\right), \ldots, \operatorname{relab}_{a_{p}}\left(H_{p}\right)\right)= \\
& \widehat{t}_{\mathbb{G}}\left(\operatorname{relab}_{a_{1}}\left(H_{1}\right), \ldots, \operatorname{relab}_{a_{p}}\left(H_{p}\right)\right) .
\end{aligned}
$$

If $t=f\left(t_{1}\right)$ where $f$ is $r e l a b_{h}$ or $a d d_{a, b}$, then the result holds because, for every $C$-graph $K$ with vertices $x_{1}, \ldots, x_{p}$, we have :

$$
\sigma\left[f(K), x_{1}, \ldots, x_{p}\right]\left(H_{1}, \ldots, H_{p}\right)=f\left(\sigma\left[K, x_{1}, \ldots, x_{p}\right]\left(H_{1}, \ldots, H_{p}\right)\right) .
$$

The equality to be proved follows then by induction.

We will denote by $t_{K}$ and $\widehat{t_{K}}$ terms associated with $K$ as above. We say that an operation $\sigma\left[K, x_{1}, \ldots, x_{p}\right]$ has width $k$ if $c w d^{\perp}(K)=k$. The operations $\oplus, \otimes, \Lambda$ and $\kappa$ have respective widths $2,2,2$ and 1 .

Proposition 3.2 : If $G \equiv \boldsymbol{v a l}(s)$ for some term $s$ in $T\left(\Sigma_{C}\right)$ whose operations have width at most $k$, then $c w d^{\perp}(G) \leq k$ and $c w d(G) \leq k+1$.

Proof : By induction on the structure of $s$, we define a term $\widetilde{s}$ in $T\left(F_{C}\right)$ such that $\boldsymbol{v a l}(\widetilde{s}) \equiv \boldsymbol{v} \boldsymbol{v a l}(s)$.

If $s=\boldsymbol{a}(w)$, then $\widetilde{s}:=s$.

If $s=\sigma\left[K, x_{1}, \ldots, x_{p}\right]\left(s_{1}, \ldots, s_{p}\right)$, then we define :

$$
\widetilde{s}:=\widehat{t_{K}}\left[\operatorname{relab}_{a_{1}}\left(\widetilde{s_{1}}\right) / u_{1}, \ldots, \text { relab }_{a_{p}}\left(\widetilde{s_{p}}\right) / u_{p}\right] \text {. }
$$

It is clear that $\operatorname{val}(\widetilde{s}) \equiv \operatorname{val}(s)$.

The set of labels used in $\widetilde{s}$ is the set of all those used in the terms $\widehat{t_{K}}$ where $\sigma\left[K, x_{1}, \ldots, x_{p}\right]$ occurs in $s$. We now bound $c w d^{\perp}(G)$. Without loss of generality, we can assume that all labels of the terms $t_{K}$ for $K$ occurring in $s$ (via some $\sigma\left[K, x_{1}, \ldots, x_{p}\right]$ ) are in a set $C$ such that $C-\{\perp\}$ has cardinality $k$. Hence $c w d^{\perp}(G) \leq k$ and $c w d(G) \leq k+1$.

\footnotetext{
${ }^{27}$ This is clear from Definition 2.1.
} 
If in $s$, all the operations of maximal width $k$ do not use $\perp$ in their definitions by terms, then $\operatorname{cwd}(G)=c w d^{\perp}(G) \leq k$.

Corollary 3.3 : Distance-hereditary graphs have clique-width at most 3

Proof : Distance-hereditary graphs are defined by means of the operations $\kappa, \Lambda, \oplus$ and $\otimes$ of width at most 2 .

This result is known from $[29,37]$ with different proofs. As the operation $\Lambda$ of width 2 needs $\perp$ in its defining term, we do not have $c w d(G)=c w d^{\perp}(G) \leq 2$. Proposition 4.9 of [36] establishes that conversely, $G$ is distance-hereditary if $c w d^{\perp}(G) \leq 2$.

\section{Split decomposition}

The split decomposition of directed and undirected graphs has been defined and studied by Cunnigham in [18]. We will formulate it in terms of graphlabelled trees, as in [27, 28], also called split-decomposition graphs in [7]. We only consider undirected graphs in this section.

\subsection{Definitions and basic facts}

Definition 4.1 : Graph-labelled trees and the graphs they describe.

We denote by $L_{T}$ the set of leaves of a tree $T$ and by $\operatorname{Inc}_{T}(v)$ the set of edges incident to a node $v$.

(a) A graph-labelled tree, denoted by $\mathcal{T}$, is a tree $T$ with at least three nodes that is equipped, for each node $v \in N_{T}$, with a connected graph $H_{v}$, called a component, and a bijection $\rho_{v}: \operatorname{Inc}_{T}(v) \rightarrow V_{H_{v}}$. The components are pairwise disjoint. We identify $u$ and the unique vertex of $H_{u}$ if $u$ is a leaf.

Figure 1 shows a graph-labelled tree with leaves $1, \ldots, 8$ and internal nodes $u, v, w, x$. The components are surrounded by elipses. The dotted lines are the edges of the tree $T$. Each of them links two vertices of two different components, and each vertex $x$ in a component $H_{v}$ is incident to one and only one "dotted edge", namely, $\rho_{v}^{-1}(x)$.

(b) The corresponding split-graph $S(\mathcal{T})$ is the union of the components together with the edges $\rho_{u}(e) \rho_{v}(e)$ for $e=u v$, (the "dotted edges" of Figure 1). A path in $S(\mathcal{T})$ is alternating if no two consecutive edges are in a same component ${ }^{28}$. Between any two vertices $x, y$ of $S(\mathcal{T})$, there is at most one alternating path. If there is one, we say that $x$ is accessible from $y$, and we precise through $z$ (or $e$ ) to indicate that this path goes through a particular vertex $z$ (or edge $e$ ). For a vertex $w$ of $S(\mathcal{T})$ belonging to a component $H_{u}$, we denote by $A(w)$

\footnotetext{
${ }^{28}$ Because of the bijections $\rho_{v}$, no two consecutive edges in a path can be tree-edges.
} 


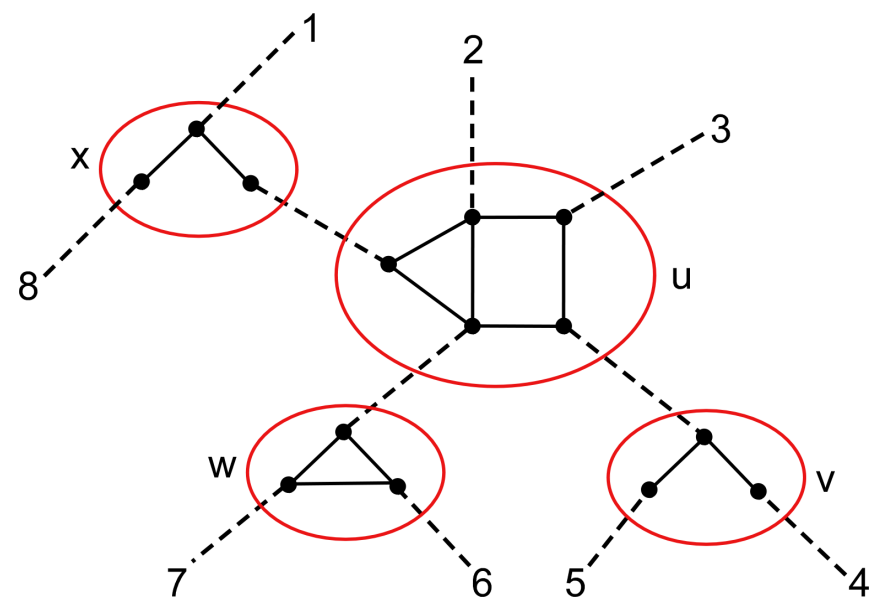

Figure 1: A graph-labelled tree $\mathcal{T}$ (cf. Definition 4.1(a).

(respectively by $P(w)$ ) the set of vertices accessible from $w$ by a nonempty alternating path whose first edge is not in $H_{u}$ (respectively, reachable from $w$ by a nonempty path in $S(\mathcal{T})$ whose first edge is not in $H_{u}$ ).

(c) The graph described by $\mathcal{T}$, denoted by $G(\mathcal{T})$, has vertex set $L_{T}$ and an edge $u v$ if and only if $u$ is accessible from $v$. It is connected ([27], Lemma 2.3) because the components are defined as connected ${ }^{29}$.

We continue to examine the graph-labelled tree $\mathcal{T}$ of Figure 1 and the associated graph $G(\mathcal{T})$ in Figure 2. There is an alternating path between leaf 7 and leaf 1 . Hence, they are adjacent vertices in $G(\mathcal{T})$. On any path between 3 and 7 , there are two consecutive edges of $H_{u}$, hence, 7 is not adjacent to 3 .

(d) Let $e=u v$ be an edge of $T$ between two internal nodes. The node-joining operation (cf. [27]) contracts this edge, hence fuses $u$ and $v$ into a single new node say $w$, giving tree $T^{\prime}$; the new component $H_{w}^{\prime}$ is defined as $H_{u} \uplus H_{v}$ minus the two vertices $\rho_{u}(e), \rho_{v}(e)$ and augmented with an edge between any vertex $x$ in $H_{u}$ and any vertex $y$ in $H_{v}$ such that $x \rho_{u}(e) \in E_{H_{u}}$ and $\rho_{v}(e) y \in E_{H_{v}}$. This graph is connected. We obtain a graph-labelled tree $\mathcal{T}^{\prime}$ (nothing else is modified from $\mathcal{T}$ ) that describes the same graph. If $\rho_{u}(e)$ has degree $r$ in $H_{u}$ and $\rho_{v}(e)$ has degree $s$ in $H_{s}$, the resulting component $H_{w}^{\prime}$ has a subgraph isomorphic to the complete bipartite graph $K_{r, s}$.

The opposite transformation is called node-splitting. It preserves also the defined graph.

Remark 4.2 : A graph $G$ consisting of a single edge is defined by a graphlabelled tree one component of which is an edge. Otherwise, a single edge compo-

\footnotetext{
${ }^{29}$ If some components are not connected, the graph defined in this way is not connected. Actually, a disconnected graph is best described as the union of its connected components. Hence, we can require that components are connected.
} 


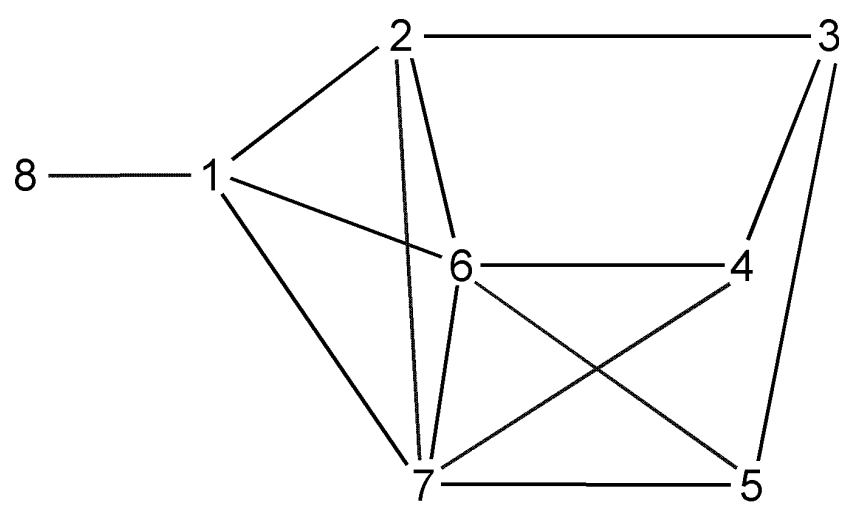

Figure 2: The graph $G(\mathcal{T})$ for $\mathcal{T}$ in Figure 1.

nent $H_{u}$ can be eliminated by a node-joining of $u$ with one of its two neighbours. This elimination being done as much as possible, the resulting tree has no node of degree 2.

However in a graph-labelled tree $\mathcal{T}$ that defines a graph with at least 2 vertices, it may be useful to insert a component consisting of a single edge (cf. Section 4.5 below) : consider an edge $u v$ of $T$, replace it by two edges $u w$ and $w v$ where $w$ is new node, and define the new component corresponding to $w$ as consisting of a single edge.

The following lemma is implicit in [14, 27]. We will generalize it for directed graphs.

If $u v$ is an edge of a tree $T$, we denote by $N_{T, u \backslash v}$ the set of nodes of the connected component of $T-u$ that contains $v$. Notation is in Definition 4.1(b).

Lemma 4.3 : Let $\mathcal{T}$ be a graph labelled tree and $G=G(\mathcal{T})$.

(1) For each vertex $x$ of $S(\mathcal{T})$, the set $A(x) \cap L_{T}$ is not empty.

(2) Let $x, y$ be distinct vertices of some component $H$. If $x y$ is an edge of $H$, there is an alternating path between any leaf in $A(x)$ and any leaf of $A(y)$. Conversely, if an alternating path links a leaf in $A(x)$ and a leaf of $A(y)$, then this path goes through $H$, and more precisely, through $x$ and $y$, and $x y \in E_{H}$. Each component is isomorphic to an induced subgraph of $G$.

(3) Let $u v$ be an edge of $T$. There is an alternating path between any leaf in $A(x)$ where $x$ is a neighbour of $\rho_{u}(u v)$ in $H_{u}$ and any leaf in $A(y)$ where $y$ is a neighbour of $\rho_{v}(u v)$ in $H_{v}$. Any such path goes through the edge $\rho_{u}(u v) \rho_{v}(u v)$.

Proof: (1) Let $x$ belong to $H_{u}$. Then $x=\rho_{u}(u v)$ for some (unique) edge $u v$ of $T$. We use induction on the cardinality of $N_{T, u \backslash v}$.

If $\left|N_{T, u \backslash v}\right|=1$, then $v$ is a leaf in $A(x)$ as $A(x)=\{v\}=\left\{\rho_{v}(u v)\right\}$. 
Otherwise, $H_{v}$ has an edge $\rho_{v}(u v) y$ and $y=\rho_{v}(v w)$ for some edge $v w$ of $T$. Then $N_{T, v \backslash w} \subset N_{T, u \backslash v}$ and $A(y) \cap L_{T} \subseteq A(x) \cap L_{T}$. The set $A(y) \cap L_{T}$ is not empty by induction, so is $A(x) \cap L_{T}$.

(2) Let $x y=\rho_{u}(u v) \rho_{u}(u w)$ be an edge of component $H_{u}$. Let $z \in A(x) \cap L_{T}$ and $z^{\prime} \in A(y) \cap L_{T}$. By connecting alternating paths between $z$ and $x$, and $z^{\prime}$ and $y$ with the edge $x y$, we get an alternating path between $z$ and $z^{\prime}$. Any such path must through $x y$ as one checks from the definitions.

For each vertex $x$ of $H_{u}$, let us choose a vertex $\widehat{x}$ of $G$ in $A(x) \cap L_{T}$. By the previous observations, the induced subgraph of $G$ whose vertices are the $\widehat{x}$ 's is isomorphic to $H_{u}$.

(3) The proof is similar to that of (2).

The following corollary illustrates these notions in the basic case of trees.

Corollary 4.4 : Let $\mathcal{T}$ be a graph-labelled tree. The graph $G(\mathcal{T})$ is a tree if and only if :

(1) each component of $\mathcal{T}$ is a tree, and

(2) if $e=u v \in E_{T}$ is not a pendant edge, then at least one of $\rho_{u}(e)$

and/or $\rho_{v}(e)$ is a leaf of, respectively, $H_{u}$ and/or $H_{v}$.

Proof : Assume $G(\mathcal{T})$ is a tree. By Lemma 4.3(2), each component of $\mathcal{T}$ is isomorphic to an induced subgraph of $G(\mathcal{T})$, hence is a tree since components are connected. For Property (2), if none of $\rho_{u}(e)$ and $\rho_{v}(e)$ is a leaf, then the nodejoining of $u$ and $v$ (Definition 4.1(d)) merges $H_{u}$ and $H_{v}$ into a component that contains a complete bipartite graph $K_{r, s}$ such that $r, s \geq 2$. This component has a cycle and $G(\mathcal{T})$ is not a tree by $(1)$.

Conversely, let $\mathcal{T}$ satisfy (1) and (2). Consider $e=u v$ satisfying Property (2) : if we apply to it the node-joining operation, the component $H_{w}^{\prime}$ created in this way is still a tree. The obtained graph-labelled tree $\mathcal{T}^{\prime}$ satisfies (1) and (2) and describes $G(\mathcal{T})$. By repeating this operation until all edges are pendant, we obtain a graph-labelled tree that defines $G(\mathcal{T})$ and that has one component that is a tree, all others being leaves. Hence $G(\mathcal{T})$ is a tree.

A tree may be defined from several nonisomorphic graph-labelled trees. A stronger condition than (2) of Corollary 4.4, namely Condition (2) of Theorem 4.6 , yields unicity, up to isomorphism, of the graph-labelled trees that define connected graphs.

Definition 4.5 : Split decompositions.

(1) A split of a graph $G$ is a bipartition of $V_{G}$ into two sets $V_{1}$ and $V_{2}$ having each at least 2 vertices, such that the edges between $V_{1}$ and $V_{2}$ induce a complete bipartite graph with at least one edge. These edges link any vertex of some set $A_{1} \subseteq V_{1}$ and any vertex of some $A_{2} \subseteq V_{2}$. Hence, $G$ is $\kappa\left(G_{1} \otimes G_{2}\right)$ where the 
induced subgraphs $G_{1}:=G\left[V_{1}\right]$ and $G_{2}:=G\left[V_{1}\right]$ have labels in $D:=\{\top, \perp\}$ in such a way that the vertices in $A_{1} \cup A_{2}$ are labelled by $\top$ and the others by $\perp$.

(2) A graph is defined as prime ${ }^{30}$ if it has at least 4 vertices and no split. The connected graphs with 3 vertices are the stars $S_{3}$ and the triangles, i.e., the graphs isomorphic to $K_{3}$. A star $S_{n}, n \geq 3$, has a center and $n-1$ adjacent vertices that are leaves: $S_{n}$ is a tree. Stars and cliques are not prime. No prime graph has less than 5 vertices. The cycles $C_{n}$ for $n \geq 5$ are prime.

Theorem 3 of [18], also proved as Theorem 2.9 in [27], states the following existence and unicity theorem.

Theorem 4.6 : Every connected graph with at least 3 vertices is $G(\mathcal{T})$ for a unique graph-labelled tree $\mathcal{T}$ such that :

(1) each component $H_{v}$ is singleton, or prime, or is a clique $K_{n}$ or a star $S_{n}$, for some $n \geq 3$,

(2) if $e=u v \in E_{T}$, then $H_{u}$ and $H_{v}$ are not both cliques, and, if they are both stars, then $\rho_{u}(e)$ and $\rho_{v}(e)$ are both centers or both leaves.

Unicity is understood up to isomorphism.

Such a graph-labelled tree is called THE split decomposition of $G$. It is canonical because of its unicity, up to isomorphism. It can be obtained from an arbitrary graph-labelled tree that defines $G$ by the node-splittings and the node-joinings of Definition 4.1(d) (see [27] for details). The resulting tree has no node of degree 2 (cf. Remark 4.2). We will also consider graph-labelled trees that need not be canonical.

Remarks 4.7 : (1) The split decomposition of a clique $K_{n}, n \geq 3$, has a unique component that is a clique. But any graph-labelled tree all components of which are cliques defines a clique. A clique $K_{n}, n \geq 3$ can be defined by a graph labelled tree all components of which are triangles or isolated vertices. By using node splitting, we can replace a component $K_{r+s+2}$ where $r, s>1$ by two components $K_{r+1}$ and $K_{s+1}$.

(2) In the split decomposition of a tree, all components are stars, and if $e=u v \in E_{T}$, then $\rho_{u}(e)$ and $\rho_{v}(e)$ are both leaves of $H_{u}$ and $H_{v}$ by Corollary 4.5 and Theorem 4.7. Every tree with at least 3 nodes can be defined by a graph-labelled tree all components of which are stars $S_{3}$ or isolated vertices. As above, this can be achieved by node-splitting.

Examples 4.8 : Distance-hereditary (DH) graphs and 3-leaf powers.

A graph-labelled tree defines a distance-hereditary graph if and only if all its components are stars and cliques, as proved in [27], Section 3.1. This article also studies particular DH graphs called 3-leaf powers. A 3-leaf power $G$ is defined as follows from a tree $S: V_{G}$ is $L_{S}$ and two vertices are adjacent if and only if they are at distance at most 3 in $S$. A graph is a 3-leaf power if and only if it

${ }^{30} \mathrm{~A}$ different notion of prime graph is used in the theory of modular decomposition, cf. [31]. 
is obtained from a tree by substitutions of cliques to its vertices [2]. It follows that a graph is a 3-leaf power if and only if it is a clique or is $G(\mathcal{T})$ for some graph-labelled tree $\mathcal{T}$ having one component, say $H_{0}$, that is a tree whereas all others are cliques (an isolated vertex is a clique $K_{1}$ ). Hence, the set $L$ of 3-leaf powers is defined, up to labels, by the two equations:

$$
\begin{aligned}
& L=K \cup \Lambda(L, L), \\
& K=\top \cup(K \otimes K) .
\end{aligned}
$$

The set $K$ is that of cliques where all vertices are alive. The equation for $L$ is derived from the first equation for rooted trees in Example 2.6(2). In the characterization of 3-leaf powers of [27], the component $H_{0}$ that is a tree is decomposed in the canonical way of Theorem 4.7 .

\subsection{Graph-labelled trees and substitution operations.}

We will use $D$-graphs, where $D:=\{\top, \perp\}$.

Definitions 4.9 : Rooted graph-labelled trees and related notions.

(a) Let $\mathcal{T}$ be a graph-labelled tree, with underlying tree $T$, not reduced to a single node. Let us select a node $r \in N_{T}$ and make it a root for $T$. We call then $\mathcal{T}$ a rooted graph-labelled tree. If $r$ is a leaf, we call it a leaf-rooted graph-labelled tree, and otherwise, a non-leaf-rooted graph-labelled tree.

(b) If $u \in N_{T}$, we $\operatorname{let}^{31} N_{u}:=\left\{x \in N_{T} \mid x \leq_{T} u\right\}$ and $V_{u}:=\left\{x \in L_{T} \mid\right.$ $\left.x \leq_{T} u\right\}$. Hence, $V_{r}=L_{T}=V_{G}$ and $V_{u}=\{u\}$ if $u \in L_{T}-\{r\}$.

(c) If $u \in N_{T}-L_{T}$ has father $w$, we say that $\rho_{u}(u w)$ is the leader of $H_{u}$, denoted by $\bar{u}$, and we define $H_{u} \backslash \bar{u}$ as the $D$-graph $H_{u}-\bar{u}$ (possibly not connected) where a vertex $x$ is alive (labelled by T) if it is adjacent to $\bar{u}$ in $H_{u}$ and is dead (labelled by $\perp$ ) otherwise. We define also $H_{r} \backslash \bar{r}:=H_{r}$, all its vertices being defined as dead. If $r$ is a leaf, then $H_{r} \backslash \bar{r}$ is undefined.

(d) If $u \in N_{T}$, we define $G_{u}$ as $G\left[V_{u}\right]$ labelled as follows :

(d.1) if $u=r$, then every vertex of $G_{u}=G$ is labelled by $\perp$,

(d.2) if $u \neq r$, a vertex $y$ of $G_{u}$ is labelled by $\top$ if it is in $A(z)$ for some neighbour $z$ (in $H_{u}$ ) of the leader of $H_{u}$; otherwise, it is labelled by $\perp$.

Note that if $r$ is a leaf and $u$ is its neighbour in $T$, then $G=G_{r}=$ $\kappa\left(\Lambda\left(\top(r), G_{u}\right)\right.$

\footnotetext{
${ }^{31} T$ will denote the rooted tree, without explicit mention of $r$; in particular, $\leq_{T}$ depends on the choice of $r$.
} 
For an example, consider the graph-labelled tree of Figure 1 in Section 4.1 with root $x$. The vertices 4 and 5 are alive in $G_{v}$, but not in $G_{u}$. The vertices $2,6,7$ are alive in $G_{u}$.

The following lemma relates graph-labelled trees and substitution operations. Note that the graphs $H_{u} \backslash \bar{u}$ depend on the root $r$ that is chosen for $T$.

Lemma 4.10 : Let $\mathcal{T}$ be a rooted graph-labelled tree that defines $G$. If $u$ in $N_{T}-L_{T}$ has sons $u_{1}, \ldots, u_{p}$, and the corresponding $p$ vertices of $H_{u} \backslash \bar{u}$ are $x_{1}, \ldots, x_{p}$ (that is, $\left.x_{i}:=\rho_{u}\left(u u_{i}\right)\right)$, then we have :

$$
G_{u}=\left(H_{u} \backslash \bar{u}\right)\left[x_{1} \leftarrow G_{u_{1}}, \ldots, x_{p} \leftarrow G_{u_{p}}\right] .
$$

Proof : Let $K:=\left(H_{u} \backslash \bar{u}\right)\left[x_{1} \leftarrow G_{u_{1}}, \ldots, x_{p} \leftarrow G_{u_{p}}\right]$.

1) The vertex set of $G_{u}$ is $V_{u}:=\left\{x \in V_{G} \mid x \leq_{T} u\right\}\left(V_{G}=L_{T}\right)$ hence, is the union the sets $V_{u_{i}}:=\left\{x \in V_{G} \mid x \leq_{T} u_{i}\right\}$ that are the vertex sets of the graphs $G_{u_{i}}$. Hence, $G_{u}$ and $K$ have the same vertices.

2) As the graphs $G_{w}$ are induced subgraphs of $G$, two vertices of $G_{u_{i}}$ are adjacent in $G_{u}$ if and only if they are in $G$ as well as in $G_{u_{i}}$, hence also in $K$.

Consider vertices $x$ of $G_{u_{i}}$ and $y$ of $G_{u_{j}}, j \neq i$. If they are adjacent in $G_{u}$, hence in $G$, they are linked by an alternating path, that must go through $H_{u}$, and not through its leader $\bar{u}$, and use its edge $\rho_{u}\left(u_{i} u\right) \rho_{u}\left(u_{j} u\right)=x_{i} x_{j}$, an edge of $H_{u} \backslash \bar{u}$. This path goes through the leader $\rho_{u_{i}}\left(u_{i} u\right)$ of $H_{u_{i}}$. Hence, $x$ is alive in $G_{u_{i}}$. Similarly, $y$ is alive in $G_{u_{j}}$. Hence $x y$ is an edge of $K$.

Conversely, if $x y \in E_{K}$, then $x_{i} x_{j}$ is an edge of $H_{u} \backslash \bar{u}$, the vertex $x$ is alive in $G_{u_{i}}$ and $y$ is alive in $G_{u_{j}}$. Going back to definitions, we have an alternating path between $x$ and $y$. Hence, $x y$ is a edge of $G$ hence of $G_{u}$.

3) If $u$ is the root $r$ and is not a leaf, then all vertices of $H_{u} \backslash \bar{u}$ are dead, hence, so are those of $K$, as well as those of $G=G_{r}$.

Otherwise, let $x$ be a vertex of $G_{u_{i}}$ that is alive. Hence, there an alterning path $P$ between $x$ and the leader $\rho_{u_{i}}\left(u_{i} u\right)$ of $H_{u_{i}}$. If $\rho_{u}\left(u_{i} u\right)=x_{i}$ is a neighbour of the leader $\bar{u}$ of $H_{u}$, then $x$ is alive in $K$. It is also in $G_{u}$ because $P$ can be extended into an alterning path from $x$ to $\bar{u}$. If $\rho_{u}\left(u_{i} u\right)=x_{i}$ is not a neighbour of $\bar{u}$, then $x$ is dead in $K$. It is also in $G_{u}$ because $P$ cannot be extended into an alterning path from $x$ to $\bar{u}$.

If $x$ is dead in $G_{u_{i}}$, then it is also in $K$ and in $G_{u}$, because otherwise, the alternating path between $x$ and $\bar{u}$ would give a path $P$ as above.

\subsection{From graph-labelled trees to grammars}

If $\mathcal{M}$ is a finite set of connected (unlabelled) graphs having at least 2 vertices, we define $\mathcal{G}(\mathcal{M})$ as the set of graphs described by graph-labelled trees whose components are in $\mathcal{M}$. These graphs are connected (Definition 4.1(c)). 
As before, $D:=\{\top, \perp\}$. For each $H \in \mathcal{M}$, we define $H_{\perp}$ as $H$ with all vertices labelled by $\perp$. We denote by $\Sigma_{\mathcal{M}}$ the set of operations $\sigma\left[H_{\perp}, x_{1}, \ldots, x_{p}\right]$ for $H \in \mathcal{M}$ and by $\Sigma_{\mathcal{M}}^{\prime}$ the set of operations $\sigma\left[H \backslash x, x_{1}, \ldots, x_{p}\right]$ for $H \in \mathcal{M}$ and $x \in V_{H}$ (cf. Definition 4.9(c) for the notation $H \backslash x$ ).

Theorem 4.11 : If $\mathcal{M}$ is a finite set of connected graphs having at least 2 vertices, then $\mathcal{G}(\mathcal{M})$ is the set $S$ defined by the two equations :

$$
\begin{aligned}
& S=\kappa(\top) \cup \cup_{\sigma \in \Sigma_{\mathcal{M}}} \sigma(U, \ldots, U) \text { and } \\
& U=\top \cup \cup_{\sigma \in \Sigma_{\mathcal{M}}^{\prime}} \sigma(U, \ldots, U) .
\end{aligned}
$$

Another grammar for $\mathcal{G}(\mathcal{M})$ consists of :

$$
S^{\prime}=\kappa(\top) \cup \kappa(\Lambda(\top, U))
$$

and the above equation that defines $U$.

Proof : Let $G$ belong to $S$. If it is a dead isolated vertex $\kappa(\top)$, it is in $\mathcal{G}(\mathcal{M})$. Otherwise, it is defined by a finite term $t=\sigma\left(t_{1}, \ldots, t_{p}\right)$ where $\sigma \in \Sigma_{\mathcal{M}}$ and $t_{1}, \ldots, t_{p}$ are terms in $T\left(\Sigma_{\mathcal{M}}^{\prime} \cup\{T\}\right)$. By Lemma 4.10 , this term represents a non-leaf-rooted graph-labelled tree $\mathcal{T}$. The component at the root is isomorphic to $H$ such that $\sigma=\sigma\left[H, x_{1}, \ldots, x_{p}\right]$. The terms $t_{1}, \ldots, t_{p}$ represent the subtrees of $T$ issued from the $p$ sons of the root. Hence, $G$ is described by a rooted graph-labelled tree with components in $\mathcal{M}$, and so, $G \in \mathcal{G}(\mathcal{M})$.

Let conversely $G \in \mathcal{G}(\mathcal{M})$. If it is a dead isolated vertex, then it is in $S$, defined by $\kappa(T)$. Otherwise it is defined by a non-leaf rooted graph-labelled tree $T$, hence, by a term $t=\sigma\left(t_{1}, \ldots, t_{p}\right)$ as above. The subtrees of $T$ issued from the sons of the root are defined by the terms $t_{1}, \ldots, t_{p}$.

The second grammar is based on leaf-rooted graph-labelled trees. The proof is similar, by the remark in Definition $4.9(\mathrm{~d})$.

The equation $W=\top \cup \Lambda(\top, U)$ with $U$ as above defines the rooted graphs in $\mathcal{G}(\mathcal{M})$ defined as those having exactly one live vertex (labelled by $\top$ ). We will apply this remark to DH graphs in Section 4.5.

\subsection{Clique-width bounds from graph-labelled trees}

Theorem 4.12 : Let $G$ be a connected graph defined by a rooted graph-labelled tree $\mathcal{T}$ such that each operation $\sigma\left[H_{u} \backslash \bar{u}, x_{1}, \ldots, x_{p}\right]$ has width at most $k \geq 2$. Then $c w d^{\perp}(G) \leq k$ and $c w d(G) \leq k+1$.

Proof: Immediate consequence of Proposition 3.2 and Theorem 4.11.

The next lemma gives an upper-bound to the widths of the terms in $T_{\perp}\left(F_{C}\right)$ that define the $D$-graphs $H_{u} \backslash \bar{u}$. 
Lemma 4.13: Let $H$ be a $D$-graph such that $c w d(H)=k$. Then $c w d^{\perp}(H) \leq$ $k+\min \left\{k,\left|V_{H}^{\text {live }}\right|,\left|V_{H}^{\text {dead }}\right|\right\}$. 2).

Proof: We have $c w d^{\perp}(H) \leq c w d^{*}(H) \leq 2 k$ by Lemma $1.3($ with $|\tau(G)| \leq$

For proving that $c w d^{\perp}(H) \leq k+\left|V_{H}^{\text {live }}\right|$, we consider a term that defines $H$ with a set $C^{\prime}$ of $k$ labels different from $\perp$. Assume that $V_{H}^{\text {live }}=\left\{x_{1}, \ldots, x_{p}\right\}$. We add new labels $c_{1}, \ldots, c_{p}$ to $C^{\prime}$ so that $c_{i}$ will only label $x_{i}$. We transform $t$ into $t^{\prime}$ accordingly. In particular, to take a typical case, if at some position in $t$ the operation $a d d_{a, b}$ adds edges between $x_{i}$, labelled at this point by $a$ (there may be other $a$-labelled vertices) and $b$-labelled vertices, then we replace it by $a d d_{c_{i}, b} \circ a d d_{a, b}$.

Hence, we can use $p+k$ labels different from $\perp$.

If $V_{H}^{\text {dead }}=\left\{x_{1}, \ldots, x_{p}\right\}$. We do a similar construction.

Theorem 4.14 : Let $G$ be defined by a graph-labelled tree $\mathcal{T}$ whose components have maximal clique-width $m$ and maximal degree $d$, then $m \leq c w d(G) \leq$ $m+\min \{m, d\}+1 \leq 2 m+1$.

Proof : The inequality $m \leq c w d(G)$ follows from Lemma 4.3(2) since cliquewidth is monotone with respect to the induced subgraph relation.

We now prove the other inequality ${ }^{32}$. For each component $H_{u}$, the number of live vertices in $H_{u} \backslash \bar{u}$ is at most $d$. Hence, Lemma 4.13 gives $c w d^{\perp}\left(H_{u} \backslash \bar{u}\right) \leq$ $m+\min \{m, d\}$ and Theorem 4.12 gives $c w d^{\perp}(G) \leq m+\min \{m, d\}$, so that $\operatorname{cwd}(G) \leq m+\min \{m, d\}+1 \leq 2 m+1$.

For an example, if $G$ is a tree that is not a star and is defined by $\mathcal{T}$ whose components are stars with three nodes, hence of clique-width 2 , we have $m=2$ and $c w d(G)=3$.

Remark 4.15: A bound based on rank-width.

Rank-width is another graph complexity measure initially defined for undirected graphs ${ }^{33}$, denoted by $r w d$. It is based on ternary trees (without root) that define layouts of the considered graphs. The DH graphs are those of rank-width 1 , as proved in [37].

Rank-width is related to clique-width by the inequalities $\operatorname{rwd}(G) \leq \operatorname{cwd}(G) \leq$ $2^{\text {rwd(G)+1 }}-1$, cf. [37]. Furthermore, if $G=G(\mathcal{T})$ for some graph-labelled tree $\mathcal{T}$, and $m$ is the maximal rank-width of a component $H_{u}$, then $\operatorname{rwd}(G)=m$ (Theorem 4.3 of [33]). Hence, if $\operatorname{cwd}\left(H_{u}\right) \leq m$ for all $u$, we get $\operatorname{rwd}(G) \leq m$ and $\operatorname{cwd}(G) \leq 2^{m+1}-1$.

Example 4.16 : Parity graphs.

\footnotetext{
${ }^{32}$ By using monadic second-order transductions [7] proves that $c w d(G)$ is bounded in terms of $m$ by a superexponential function.

${ }^{33}$ The extension to directed graphs is in [34].
} 
A graph is a parity graph if for any two vertices, the induced paths joining them have the same parity. Bipartite graphs and DH graphs are parity graphs. The article [5] establishes that the parity graphs are the graphs having a split decomposition whose components are cliques and bipartite graphs. We do not obtain a finite grammar as bipartite graphs, whence also parity graphs, have unbounded clique-width.

\subsection{Unambigous grammars for cographs and distance-hereditary graphs}

We first examine some of the operations $\sigma\left[H, x_{1}, \ldots, x_{p}\right]$ that arise in split decompositions.

Observation 4.17 : Substitution operations related to split decompositions.

Case 1: $H$ is a clique $K_{p}, p \geq 2$ whose vertices $x_{1}, \ldots, x_{p}$ are all alive. We have :

$$
\sigma\left[K_{p}, x_{1}, \ldots, x_{p}\right]\left(G_{1}, \ldots, G_{p}\right)=G_{1} \otimes \ldots \otimes G_{p} .
$$

Case 2: $H=S_{p} \backslash x_{p}$ where $p \geq 3$ and $S_{p}$ has center $x_{1}$ that is alive, all other vertices being dead. We have :

$$
\begin{aligned}
& \left.\left.\left.\sigma\left[S_{p} \backslash x_{p}, x_{1}, \ldots, x_{p-1}\right]\left(G_{1}, \ldots, G_{p-1}\right)=\Lambda\left(\Lambda\left(\ldots \Lambda\left(G_{1}, G_{2}\right), G_{3}\right), \ldots, G_{p-1}\right)\right) \ldots\right)\right) \\
& =\Lambda\left(G_{1}, G_{2} \oplus G_{3} \oplus \ldots \oplus G_{p-1}\right) \text { by Equality (5) of Definition } 2.5 .
\end{aligned}
$$

Case 3: $H=S_{p} \backslash x_{p}$ where $p \geq 3, S_{p}$ has center $x_{p}$ and all vertices of $S_{p} \backslash x_{p}$ are alive. Then we have :

$$
\sigma\left[S_{p} \backslash x_{p}, x_{1}, \ldots, x_{p-1}\right]\left(G_{1}, \ldots, G_{p-1}\right)=G_{1} \oplus \ldots \oplus G_{p-1} .
$$

Constructions 4.18 : Grammars for DH graphs revisited.

A connected DH graph without live vertices is defined by a graph-labelled tree $\mathcal{T}$ whose components are stars, cliques and single vertices. By rooting $T$ at a leaf, we obtain from Theorem 4.11 the following grammar, where $S$ defines the connected DH graphs :

$$
\begin{aligned}
& S=\kappa(\top) \cup \kappa(\Lambda(\top, U)), \\
& U=\top \cup(U \oplus U) \cup(U \otimes U) \cup \Lambda(U, U) .
\end{aligned}
$$

Here is an alternative construction, where the chosen root is not a leaf. By means of node splittings (Definition 4.1(d)), we can transform $\mathcal{T}$ as above into a graph-labelled tree whose components are stars $S_{3}$, triangles $K_{3}$, together with one component ${ }^{34} K_{2}$ : for $n \geq 4$, a component (isomorphic to) $K_{n}$ can be split

${ }^{34}$ By Remark 4.2 . 
into $K_{3}$ and $K_{n-1}$, and a component $S_{n}$ can be split into $S_{3}$ and $S_{n-1}$ where the center of $S_{3}$ is linked to a leaf of $S_{n-1}$.

Let us take as root the component $K_{2}$. We obtain from Theorem 4.11 the following equations :

$$
\begin{aligned}
& S=\kappa(\top) \cup \kappa(U \otimes U), \\
& U=\top \cup(U \oplus U) \cup(U \otimes U) \cup \Lambda(U, U),
\end{aligned}
$$

that are the two equations of Proposition 2.7(2).

The equations of Proposition 2.7 can be used to generate DH graphs having a given number $n$ of vertices, but not, at least immediately, with equal probability for each fixed $n$. The reason is that because of the associativity and commuttivity of $\oplus$ and $\otimes$, and also because of Equality (5) of Definition 2.5, this grammar is ambigous. For the same reason, it cannot be used for counting ${ }^{35}$ the number of DH graphs having $n$ vertices. However, it can be transformed so as to allow that.

Construction 4.19: An unambigous grammar for cographs.

Cographs are $\mathrm{DH}$ and defined by the equation :

$$
C=\top \cup(C \oplus C) \cup C \otimes C .
$$

They have a canonical description as follows, where $C_{\otimes}$ (resp. $C_{\oplus}$ ) denotes the set of connected (resp. disconnected) cographs with at least two vertices. A ( $T$-labelled, abstract) cograph $G$ is :

a single vertex $\top$,

or it is connected and of the form $G_{1} \otimes \ldots \otimes G_{p}, p \geq 2$, where $G_{1}, \ldots, G_{p} \in$ $C_{\oplus} \uplus\{\top\}$,

or it is disconnected and of the form $G_{1} \oplus \ldots \oplus G_{p}, p \geq 2$, where $G_{1}, \ldots, G_{p} \in$ $C_{\otimes} \uplus\{\top\}$.

The corresponding term is (or represents) the (canonical) modular decomposition of $G$ [31]. For a finite or countable set $\mathcal{L}$ of labelled graphs, we define:

$\oplus_{\geq 2}(\mathcal{L})$ as the set of labelled graphs $G_{1} \oplus \ldots \oplus G_{p}$ and

$\otimes_{\geq 2}(\mathcal{L})$ as the set of labelled graphs $G_{1} \otimes \ldots \otimes G_{p}$,

where, in both cases, $p \geq 2$ and $G_{1}, \ldots, G_{p} \in \mathcal{L}$.

With these "metaoperations", we can define cographs by the three equations:

$$
\begin{aligned}
& C=\top \cup C_{\oplus} \cup C_{\otimes}, \\
& C_{\oplus}=\oplus \geq 2 \\
& C_{\otimes}=\otimes_{\geq 2}\left(\top \cup C_{\otimes}\right),
\end{aligned}
$$

\footnotetext{
${ }^{35}$ The term enumerating creates confusion with the problem of listing graphs or configurations in graphs.
} 
These three equations form an unambigous grammar because of the unicity of the decomposition recalled above, and because $G_{1} \oplus \ldots \oplus G_{p}=G_{\pi(1)} \oplus \ldots \oplus G_{\pi(p)}$ for any permutation $\pi$ of the indices, and similarly for $\otimes$. Hence, $\oplus_{\geq 2}$ can be seen as an operation of variable arity extended to sets. One obtains a bijection of the set connected (abstract) cographs with the rooted trees whose internal nodes have at least two sons. The sons of a node form a set and not a sequence. These trees, called hierarchies, have been used in [38] to evaluate the number of cographs of a given size.

Construction 4.20 : An unambigous grammar for DH graphs.

For distance-hereditary graphs, the situation is similar, by using canonical split decompositions. The grammars given in Construction 4.18 are ambigous. We can obtain an unambigous one for rooted and connected DH graphs. Rooted means that one vertex is distinguished as alive and all others are dead. We only generate such graphs having at least 3 vertices.

Theorem 4.6 and Example 4.8 yield the following description that we give as a grammar written with the two above metaoperations:

$$
\begin{aligned}
& D=\Lambda\left(\top, D_{\otimes} \cup D_{\oplus} \cup D_{\Lambda}\right), \\
& D_{\otimes}=\otimes_{\geq 2}\left(\top \cup D_{\oplus} \cup D_{\Lambda}\right), \\
& D_{\oplus}=\oplus_{\geq 2}\left(\top \cup D_{\otimes} \cup D_{\Lambda}\right), \\
& D_{\Lambda}=\Lambda\left(J, D_{\oplus} \cup \Lambda\left(J, \top \cup D_{\otimes} \cup D_{\Lambda}\right),\right. \\
& J=\top \cup D_{\oplus} \cup D_{\otimes} .
\end{aligned}
$$

The equation $D=\Lambda\left(\top, D_{\otimes} \cup D_{\oplus} \cup D_{\Lambda}\right)$ defines the root by $\top$ and $D_{\otimes} \cup D_{\oplus} \cup$ $D_{\Lambda}$ correspond to the three types of components $H_{u}$ of its son $u$, respectively Cases 1,3 and 2 in Observations 4.17.

The equation $D_{\otimes}=\otimes_{\geq 2}\left(\top \cup D_{\oplus} \cup D_{\Lambda}\right)$ corresponds to a component that is a clique (cf. Case 1). The righthand side does not include $D_{\otimes}$ because two clique components cannot be neighbour in a canonical split decomposition.

The equation $D_{\oplus}=\oplus_{\geq 2}\left(\top \cup D_{\otimes} \cup D_{\Lambda}\right)$ corresponds to a star component whose center is the leader. The righthand side does not include $D_{\oplus}$ because two star components cannot be linked by their centers.

The rules for $D_{\Lambda}$ correspond a star component $H_{u}$ whose center is not the leader. The set $J$ corresponds to the son of $u$ linked to the center. It does not does not include $D_{\Lambda}$ because two star components cannot be linked by two leaves. The term $\Lambda\left(J, \top \cup D_{\otimes} \cup D_{\Lambda}\right)$ corresponds to a star $S_{3}=H_{u}$, and the term $\Lambda\left(J, D_{\oplus}\right)$ to larger stars.

In this grammar, an equation like $D_{\otimes}=\otimes_{\geq 2}\left(\top \cup D_{\oplus} \cup D_{\Lambda}\right)$ creates no ambiguity because the three sets $\{\top\}, D_{\oplus}$ and $D_{\Lambda}$ are disjoint and the metaoperation $\otimes_{\geq 2}$ avoids the ambiguities created by the associativity and commutativity of $\otimes$. That the full grammar is unambigous follows from the unicity part of Theorem 4.6 .

These rules appear in Appendix A of [4], written so as to yield corresponding generating functions. We recall that unambiguity is essential for counting purposes, and also for random generation (cf. [25]). 
Distance-hereditary graphs without root (without any vertex distinguished as the unique live one) are obtained by adding the rule $E=\kappa(D)$, but the resulting grammar becomes ambigous because the derivation trees corresponding to different roots are different. A more complex grammar that is appropriate for counting the $\mathrm{DH}$ graphs without root is given in [4]. It uses rules with substractions so as to avoid double counting. (They apply the equality $|A \cup B|=|A|+|B|-|A \cap B|$.) This article also handles 3-leaf powers (cf. Example 4.8) in a similar way.

\section{Directed graphs}

We now extend our results to directed graphs. Cunnigham defines a canonical split decomposition for the directed graphs that are strongly connected (Theorem 1 in [18]). An undirected graph can be seen as a directed one where each arc (directed edge) has an opposite one. It is connected if and only if the corresponding directed graph is strongly connected. Hence, Theorem 4.7 is a special case of a more general one for directed graphs ${ }^{36}$.

We will use graph-labelled trees and split decomposition graphs as in [7]. In order to extend to directed graphs the results of Section 4, we will revise the notion of substitution of Section 2 for graphs with labels that encode the directions of arcs. The set of live vertices is partitioned into three sets, designated by the tags $T,+,-$, attached to the labels of the sets $C$ used to construct graphs with the clique-width operations of Definition 1.2.

We study directed graphs. However, the graphs representing graph-labelled trees will have undirected edges as well as arcs.

\subsection{Substitution}

Definition 5.1 : Substitutions of directed graphs to vertices

We let $D:=\{\perp,+,-, \top\}$ be ordered in such a way that $\perp<+<\top$, $\perp<-<\top$, and + and - are incomparable. Let $K$ be a $D$-graph with vertex set $\left\{x_{1}, \ldots, x_{p}\right\}$ and $H_{1}, \ldots, H_{p}$ be pairwise disjoint $D$-graphs, that are disjoint from $K$. We define a $D$-graph $G:=K\left[x_{1} \leftarrow H_{1}, \ldots, x_{p} \leftarrow H_{p}\right]$ :

$$
\begin{aligned}
& V_{G}:=V_{H_{1}} \uplus \ldots \uplus V_{H_{p}}, \\
& \pi_{G}(v):=\inf \left\{\pi_{H_{i}}(v), \pi_{K}\left(x_{i}\right)\right\} \text { if } v \in V_{H_{i}} .
\end{aligned}
$$

Its arcs are as follows, for $u, v \in V_{G}$ (they do not depend on $\pi_{K}$ ) :

\footnotetext{
${ }^{36}$ We thought better to begin with undirected graphs, because the formal setting is much simpler and most of Graph Structure Theory and Graph Algorithmics is devoted to undirected graphs.
} 


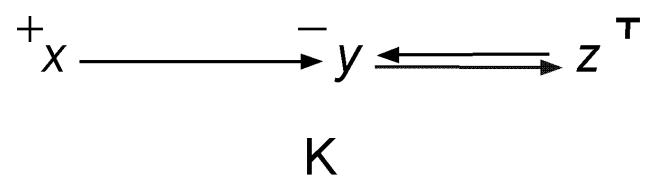

$0 \perp$

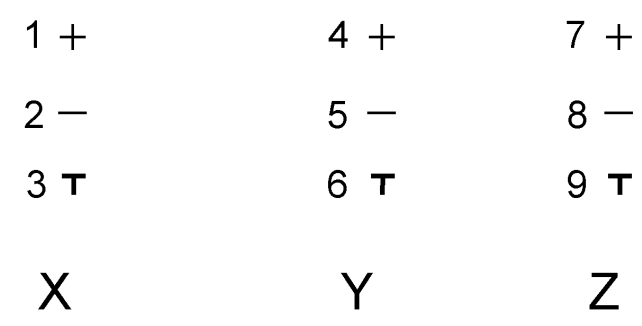

Figure 3: Graphs from Example 5.2

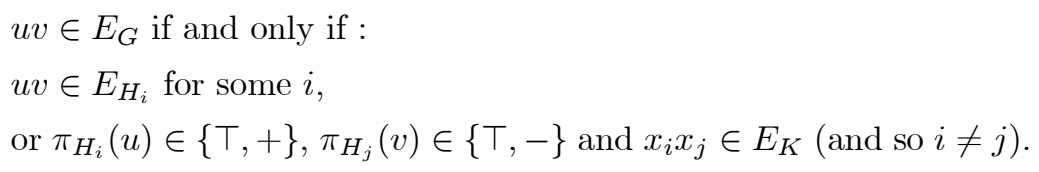

Lemma 5.13 below motivates this definition. If we consider an undirected graph as a directed graph where each arc has an opposite one, and whose vertices are labelled by $\perp$ or $T$, then, Definition 5.1 gives the same notion of substitution as Definition 2.1.

Example 5.2 : Let $K$ have vertices $x, y$ and $z$, respectively labelled by,+and $\top$, and $\operatorname{arcs} x y, y z$ and $z y$. Let $X$ be the edgeless graph with vertices $0,1,2,3$ labelled respectively by $\perp,+,-, \top$. Let similarly $Y$ have vertices $4,5,6$ labelled by,,$+- \top$ and $Z$, vertices $7,8,9$ labelled by,,$+- \top$. The graphs $K, X, Y, Z$ and $G:=K[x \leftarrow X, y \leftarrow Y, z \leftarrow Z]$ are shown in Figures 3 and 4 . The labels of vertices $0,1,5,7,8$ and 9 are as in $X, Y$ and $Z$. Those of $2,3,4$ and 6 are respectively $\perp=\inf \{+,-\},+=\inf \{+, \top\}, \perp=\inf \{+,-\}$ and $-=\inf \{-, \top\}$, cf. Definition 5.1(a).

We obtain graph operations, as in Section 2, that we will use to describe the directed graphs defined by graph-labelled trees.

Definition 5.3 : Graph operations based on substitution.

For each $D$-graph $K$ with vertex set enumerated as $\left\{x_{1}, \ldots, x_{p}\right\}$, we define as follows a $p$-ary operation on $D$-graphs denoted by $\sigma\left[K, x_{1}, \ldots, x_{p}\right]$ :

$$
\sigma\left[K, x_{1}, \ldots, x_{p}\right]\left(H_{1}, \ldots, H_{p}\right):=K\left[x_{1} \leftarrow H_{1}, \ldots, x_{p} \leftarrow H_{p}\right]
$$




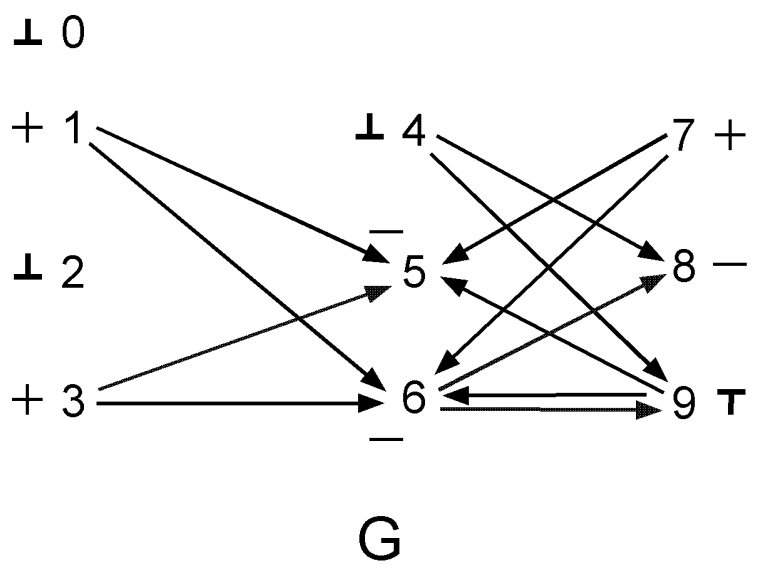

Figure 4: Graph $G$ of Example 5.2.

where $H_{1}, \ldots, H_{p}$ are pairwise disjoint and disjoint from $K$. If they are not, we replace them by isomorphic copies, so that $\sigma\left[K, x_{1}, \ldots, x_{p}\right]$ becomes a $p$-ary operation on abstract $D$-graphs.

If we extend Definition 5.1, so that $K$ can have other vertices than $x_{1}, \ldots, x_{p}$, then Proposition 2.2 is still valid.

We will express substitutions in terms of clique-width operations. For directed graphs, we use the operations $\overrightarrow{a d d}_{a, b}$ that create arcs from $a$-labelled vertices to $b$-labelled ones. Our objective is to bound the clique-width of $G:=$ $K\left[x_{1} \leftarrow H_{1}, \ldots, x_{p} \leftarrow H_{p}\right]$ as $O\left(\max \left\{c w d(K), \operatorname{cwd}\left(H_{1}\right), \ldots, c w d\left(H_{p}\right)\right\}\right)$.

Definitions 5.4 : Clique-width operations and substitution.

The set $D:=\{\perp, \top,+,-\}$ is ordered by Definition 5.1. Let $K, H_{1}, \ldots, H_{p}$ be $D$-graphs and $G:=K\left[x_{1} \leftarrow H_{1}, \ldots, x_{p} \leftarrow H_{p}\right]$.

We assume that the graphs $K, H_{1}, \ldots, H_{p}$ are defined, up to their labels in $D$, by terms in $T\left(F_{C}\right)$ where $C \cap D=\emptyset$. We define $C^{\#}:=\{(a, \alpha, \beta) \mid a \in$ $C, \alpha, \beta \in D, \beta \leq \alpha\}$ and $f: C^{\#} \rightarrow D$ such that $f((a, \alpha, \beta)):=\beta$ for all $(a, \alpha, \beta)$ in $C^{\#}$.

We will define the $D$-graph $G$ by a term $t_{G}$ in $T\left(F_{C^{\#}}\right)$ in such a way that $G=\operatorname{relab}_{f}\left(\boldsymbol{v a l}\left(t_{G}\right)\right)$.

Assume that $K=\boldsymbol{v a l}\left(t_{K}\right)$ for $t_{K} \in T\left(F_{C}\right)$. Let $x_{1}, \ldots, x_{p}$ be the vertices of $K$. Each vertex $x_{i}$ is defined by a nullary symbol $\boldsymbol{a}_{\boldsymbol{i}}\left(x_{i}\right)$ in $t_{K}$. We construct a term $\widehat{t_{K}}$ in $T\left(F_{C \#},\left\{u_{1}, \ldots, u_{p}\right\}\right)$ as follows :

- we replace each $\boldsymbol{a}_{\boldsymbol{i}}\left(x_{i}\right)$ by the variable $u_{i}$,

- we replace each operation rela $_{h}$ by rela $b_{\widehat{h}}$ where $\widehat{h}((a, \alpha, \beta)):=(h(a), \alpha, \beta)$ for all $(a, \alpha, \beta) \in C^{\#}$, 
- we replace each operation $\overrightarrow{a d d}_{a, b}$ by the composition (in any order) of the operations $\overrightarrow{a d d}_{(a, \alpha, \beta),\left(b, \alpha^{\prime}, \beta^{\prime}\right)}$ such that $\alpha \in\{\top,+\}, \alpha^{\prime} \in\{\top,-\}$ and $\beta, \beta^{\prime} \in D$, $\beta \leq \alpha, \beta^{\prime} \leq \alpha^{\prime}$.

Furthermore, for each $i:=1, \ldots, p$, we define $h_{i}: C^{\#} \rightarrow C^{\#}$ by $h_{i}((a, \alpha, \beta)):=$ $\left(a_{i}, \beta, \inf \left\{\beta, \pi_{K}\left(x_{i}\right)\right\}\right)$ for $(a, \alpha, \beta) \in C^{\#}$

Lemma 5.5 : Let $K, H_{1}, \ldots, H_{p}, G, t_{K}$ and $\widehat{t_{K}}$ be as in Definition 5.4. Assume that for each $i$, we have a term $t_{i} \in T\left(F_{C \#}\right)$ such that $H_{i}=\operatorname{relab}_{f}\left(\boldsymbol{v a l}\left(t_{i}\right)\right)$. Then :

$K\left[x_{1} \leftarrow H_{1}, \ldots, x_{p} \leftarrow H_{p}\right]=\operatorname{relab}_{f}\left(\boldsymbol{v a l}\left(\widehat{t_{K}}\left[\operatorname{relab}_{h_{1}}\left(t_{1}\right) / u_{1}, \ldots, \operatorname{relab}_{h_{p}}\left(t_{p}\right) / u_{p}\right]\right)\right)$.

Proof : Let

$$
\begin{aligned}
& G:=K\left[x_{1} \leftarrow H_{1}, \ldots, x_{p} \leftarrow H_{p}\right] \text { and } \\
& G^{\prime}:=\operatorname{relab}_{f}\left(\boldsymbol{v a l}\left(\widehat{t_{K}}\left[\operatorname{relab}_{h_{1}}\left(t_{1}\right) / u_{1}, \ldots, \operatorname{relab}_{h_{p}}\left(t_{p}\right) / u_{p}\right]\right)\right) .
\end{aligned}
$$

These two graphs have the same vertex set, $V_{H_{1}} \uplus \ldots \uplus V_{H_{p}}$. We compare their labels.

Let $u \in V_{H_{i}}$ and $(a, \alpha, \beta)$ be its label in $\boldsymbol{v a l}\left(t_{i}\right)$. Its label is $\beta$ in $H_{i}$, and it is $\left(a_{i}, \beta, \inf \left\{\beta, \pi_{K}\left(x_{i}\right)\right\}\right)$ in $\operatorname{relab}_{h_{i}}\left(t_{i}\right)$. Its label in $G^{\prime}$ is thus $\inf \left\{\beta, \pi_{K}\left(x_{i}\right)\right\}$ because the relabellings in $\widehat{t_{K}}$ do not modify the third components of labels. It is the same in $G$ by Definition 5.1.

We now compare the $\operatorname{arcs}$ of $G$ and $G^{\prime}$.

Case $1: u, v \in V_{H_{i}}$. If $u v \in E_{H_{i}}$, it is also an arc of $G$ and of $G^{\prime}$. If $u v \notin E_{H_{i}}$, it is not an arc of $G$ either. And it is not an arc of $G^{\prime}$ because the labels of $u$ and $v$ have the same first components, namely $a_{i}$, in relab $b_{h_{i}}\left(t_{i}\right)$ and the relabellings in $\widehat{t_{K}}$ maintain this equality. Hence, no arc between $u$ and $v$ is created by the operations $\overrightarrow{a d d}_{(a, \alpha, \beta),\left(b, \alpha^{\prime}, \beta^{\prime}\right)}$ of $\widehat{t}_{K}$ (where we must have $a \neq b$ ). Hence, $u v \notin E_{G^{\prime}}$.

Case 2 : $u \in V_{H_{i}}, v \in V_{H_{j}}, i \neq j$. If $u v \in E_{G}$, then $x_{i} x_{j}$ is an arc of $K$ and the labels of $u$ and $v$ in $H_{i}$ and $H_{j}$ are respectively $\alpha \in\{\top,+\}$ and $\alpha^{\prime} \in\{\top,-\}$ by Definition 5.1. Let us now consider $G^{\prime}$. At some position $p$ in $t_{K}$ the arc $x_{i} x_{j}$ is created by an operation $\overrightarrow{a d d}_{a, b}$.

In $\boldsymbol{v a l}\left(\operatorname{relab}_{h_{i}}\left(t_{i}\right)\right)$ and $\boldsymbol{v a l}\left(\operatorname{relab}_{h_{j}}\left(t_{j}\right)\right)$, the labels of $u$ and $v$ are respectively $\left(a_{i}, \alpha, \beta\right)$ and $\left(a_{j}, \alpha^{\prime}, \beta^{\prime}\right)$ for some $\beta$ and $\beta^{\prime}$, and $a_{i}$ and $a_{j}$ are relabelled in $t_{K}$ into $a$ and $b$ at position $p$. The labels of $u$ and $v$ are thus $(a, \alpha, \beta)$ and $\left(b, \alpha^{\prime}, \beta^{\prime}\right)$ in $\widehat{t_{K}}$ at the place corresponding to $p$ in the construction of $\widehat{t_{K}}$ from $t_{K}\left(\overrightarrow{a d d}_{a, b}\right.$ is replaced by a composition of arc additions). Hence, $u v$ is an arc of $G^{\prime}$, created by $\overrightarrow{a d d}_{(a, \alpha, \beta),\left(b, \alpha^{\prime}, \beta^{\prime}\right)}$.

Hence every arc of $G$ is one of $G^{\prime}$. The proof is similar in the other direction. Hence, $G=G^{\prime}$.

No label $(a, \perp, \perp)$ occurs in an arc addition operation of $\widehat{t_{K}}$. Furthermore, $f((a, \perp, \perp))=\perp$ for each $a \in C$. Hence, all labels $(a, \perp, \perp)$ can be replaced by the unique label $(c, \perp, \perp)$ for some fixed $c \in C$. It follows that $\widehat{t_{K}}$ and the relabellings $h_{i}$ only use the following $8|C|+1$ labels : 


$$
\begin{aligned}
& (a, \top, \top),(a, \top,+),(a, \top,-),(a, \top, \perp),(a,+,+),(a,+, \perp), \\
& (a,-,-),(a,-, \perp) \text { for all } a \in C \text { and }(c, \perp, \perp) .
\end{aligned}
$$

By using this remark, we obtain:

Proposition 5.6 : If a graph $G$ is defined up to vertex labels by a composition of operations $\sigma\left[K, x_{1}, \ldots, x_{p}\right]$ such that each graph $K$ has clique-width at most $k$, then, $\operatorname{cwd}(G) \leq 8 k+1$.

Proof: Let $G$ be defined by a term over nullary symbols and operations $\sigma\left[K, x_{1}, \ldots, x_{p}\right]$ such that $\operatorname{cwd}(K) \leq k$. The terms $t_{K}$ can be written with the labels of a set $C$ of $k$ labels. By composing the terms $\widehat{t_{K}}$ and the relabellings $h_{i}$ according to Lemma 5.5 , we obtain a term in $T\left(F_{C^{\#}}\right)$ that defines $G$ by using at most $8 k+1$ labels. Hence, $\operatorname{cwd}(G) \leq 8 k+1$.

\subsection{Directed graph-labelled trees.}

Definition 5.7 : Graph-labelled trees describing directed graphs.

In the following definition, unspecified notions and notation are as in Definition 4.1. Graph labelled-trees will be represented by graphs with (directed) arcs and (undireceted) edges. Let us consider an edge as a pair of opposite arcs. Then a directed path or walk ${ }^{37}$ can go through an edge, that is, through one of the two arcs of this edge.

(a) A directed graph-labelled tree, denoted by $\mathcal{T}$ is an undirected tree $T$ with at least 3 nodes, that is equipped, for each node $v \in N_{T}$, with a directed graph $H_{v}$, called a component, and a bijection $\rho_{v}: \operatorname{Inc}_{T}(v) \rightarrow V_{H_{v}}$. Components are pairwise disjoint and need not be connected, see Example 5.8(2) below. If $v$ is a leaf, then $H_{v}$ has a single vertex that we identify with $v$.

(b) We define $S(\mathcal{T})$ as the graph consisting of the union of the components augmented with the (undirected) edges $\rho_{u}(e) \rho_{v}(e)$ for $e=u v$, (cf. Figure 5, where the edges $\rho_{u}(e) \rho_{v}(e)$ are shown by dotted lines). The arcs are inside the components. A directed path or walk in $S(\mathcal{T})$ is alternating if no two consecutive arcs are in a same component. Hence, in a directed path, arcs alternate with edges. In a directed walk, an edge can be traversed consecutively several times.

There is at most one alternating path from a vertex $x$ to a vertex $y$, but there may also exist one from $y$ to $x$. This is the case if and only if each arc $z z^{\prime}$ on this path inside a component has an opposite $\operatorname{arc} z^{\prime} z$ (we are considering paths, not walks).

In view of Proposition 5.9, for a vertex $x$ in a component $H_{u}$, we define $A^{-}(x)$ as the set of vertices $y$ of $S(\mathcal{T})$ accessible from $x$ by an alternating path (from $x$ to $y$ ) whose first edge or arc is not in $H_{u}$, and $A^{+}(x)$ as the set of vertices $w$ of $S(\mathcal{T})$ such that there is an alternating path from $w$ to $x$ whose last edge or arc is not in $H_{u}$.

\footnotetext{
${ }^{37} \mathrm{~A}$ walk is like a path but it can go several times through a vertex or an arc.
} 


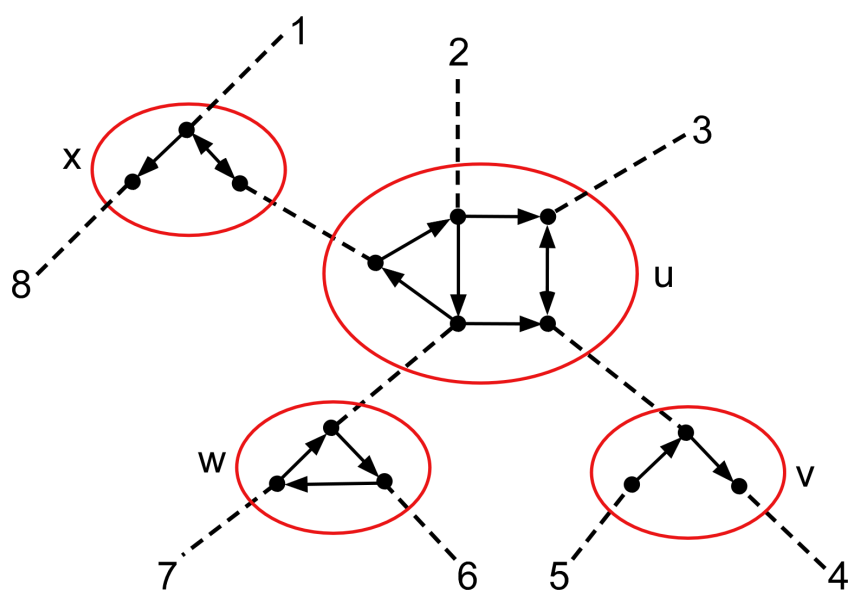

Figure 5: The directed graph-labelled tree $T$ of Example 5.8(1).

(c) The graph described by $\mathcal{T}$, denoted by $G(\mathcal{T})$, has vertex set $L_{T}$ (the set of leaves of $T$ ) and an arc $u v$ if and only if there is an alternating path from $u$ to $v$. If there is a directed path $u_{1} \rightarrow u_{2} \ldots \rightarrow u_{p}$ in $G(\mathcal{T})$, the concatenation of the alternating paths corresponding to the arcs $u_{i} u_{i+1}$ forms an alternating walk from $u_{1}$ to $u_{p}$.

(d) The node-joining operation ${ }^{38}$ (called elimination of an edge $e$ of $T$ in [7]) is defined as follows. If $e=u v$ is an edge between two internal nodes of $T$, its contraction fuses $u$ and $v$ into a single node say $w$, giving tree $T^{\prime}$, and replaces the graphs $H_{u}$ and $H_{v}$ by $H_{w}^{\prime}:=\left(H_{u} \uplus H_{v}\right)-\left\{\rho_{u}(e), \rho_{v}(e)\right\}$ augmented with an arc from any vertex $x$ in $H_{u}$ to any vertex $y$ in $H_{v}$ such that $x \rho_{u}(e) \in E_{H_{u}}$ and $\rho_{v}(e) y \in E_{H_{v}}$. We obtain a directed graph-labelled tree $\mathcal{T}^{\prime}$ that describes the same graph : this is easy to check by considering alternating paths. By iterating as much as possible this elimination step, we obtain a star whose central component is isomorphic to $G(\mathcal{T})$.

The opposite transformation called node-splitting also preserves the defined graph.

Theorem 2 of [18] defines canonical decompositions for strongly connected graphs whose components are cliques, stars and particular graphs called cycles of transitive tournaments that have clique-width at most 4 by Proposition 4.16 of [7]. We will not use this difficult notion. We will describe directed, possibly disconnected graphs by directed graph-labelled trees, either canonical or not.

Examples 5.8 : (1) Figures 5 shows a directed graph-labelled tree $\mathcal{T}$ and Figure 6 the graph $G(\mathcal{T})$. The double arrows in the components $H_{x}$ and $H_{u}$

\footnotetext{
${ }^{38}$ Similar to that in Definition 4.1(d).
} 


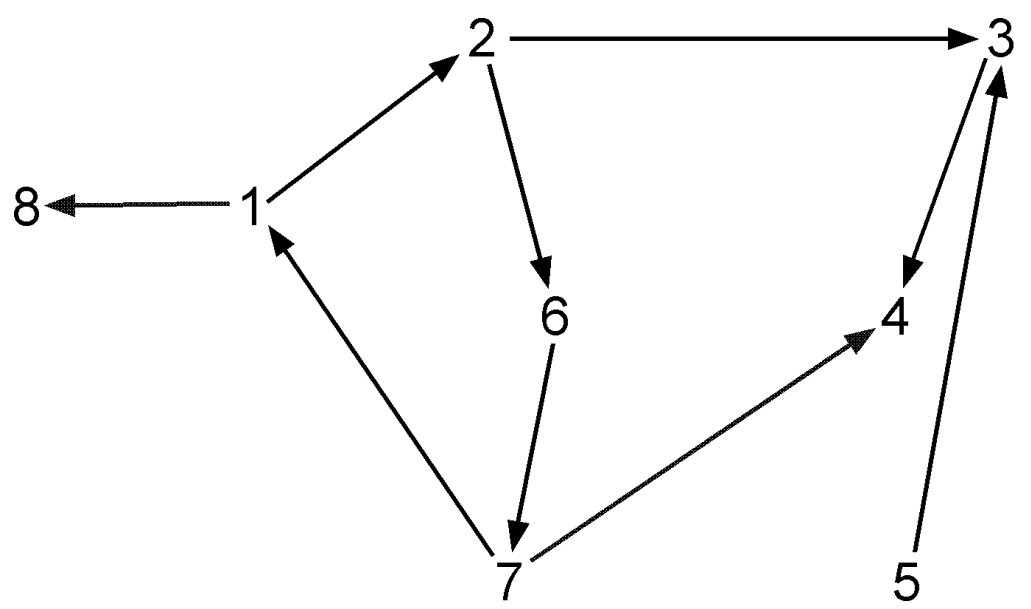

Figure 6: The graph defined by $\mathcal{T}$ of Figure 5 .

indicate pairs of opposite arcs. For a comparison with Figure 1, there is here no alternating path between 2 and 7, in either direction. Hence, these two vertices are not adjacent in $G(\mathcal{T})$.

(2) A directed graph-labelled tree may define a disconnected graph although its components are connected. As a small example, consider $S(\mathcal{T})$ defined as $x-z^{\prime} \longrightarrow z-u \longleftarrow u^{\prime}-y$, whose internal components are $z^{\prime} \longrightarrow z$ and $u \longleftarrow u^{\prime}$. (The undirected edges between components are $x-z^{\prime}, z-u$ and $\left.u^{\prime}-y\right)$. Then $G(\mathcal{T})$ consists of the two isolated vertices $x$ and $y$. Eliminating the edge $z-u$ yields a component consisting of two isolated vertices $u^{\prime}$ and $z^{\prime}$. Because of this observation, it is pointless to require that components be connected.

Proposition 5.9 : Let $G$ be defined by a directed graph-labelled tree $\mathcal{T}$ whose components are strongly connected.

(1) For each vertex $x$ of $S(\mathcal{T})$, we have $A^{+}(x) \cap L_{T} \neq \emptyset$ and $A^{-}(x) \cap L_{T}$ $\neq \emptyset$.

(2) If $x y$ is an arc of $H_{u}$, then $z z^{\prime} \in E_{G}$ for all $z \in A^{+}(x) \cap L_{T}$ and $z^{\prime} \in A^{-}(y) \cap L_{T}$. Conversely, if $x=\rho_{u}(u v)$ and $y=\rho_{u}(u w)$ are distinct vertices of $H_{u}$, if $z \in L_{T} \cap N_{T, u \backslash v}, z^{\prime} \in L_{T} \cap N_{T, u \backslash w}$ and $z z^{\prime} \in E_{G}$, then $z \in A^{+}(x) \cap L_{T}$, $z^{\prime} \in A^{-}(y) \cap L_{T}$ and $x y$ is an arc of $H_{u}$.

(3) $G$ is strongly connected.

Note that $T$ has at least two leaves and so, that $G$ has at least two vertices.

Proof : (1) Let $x$ be a vertex of a component $H_{u}$. Hence, $x=\rho_{u}(u v)$ for some node $v$. We use induction on the cardinality of $N_{T, u \backslash v}$ (the set nodes of $T$ reachable from by a path going through $v$; cf. Lemma 4.3). If it is 1 , then $v$ is a leaf and $A^{+}(x) \cap L_{T}=A^{-}(x) \cap L_{T}=\{v\}$. 
Otherwise, we have in $H_{v}$ an arc $z y$ such that $y=\rho_{v}(u v)$ and $z=\rho_{v}(v w)$ for some edge $v w$ of $T$. We have $N_{T, v \backslash w} \subset N_{T, u \backslash v}$, hence, by induction, $A^{+}(z) \cap L_{T}$ $\neq \emptyset$. As $A^{+}(z) \cap L_{T} \subseteq A^{+}(x) \cap L_{T}$ we have $A^{+}(x) \cap L_{T} \neq \emptyset$. The proof that $A^{-}(x) \cap L_{T} \neq \emptyset$ is similar ${ }^{39}$.

(2) Just consider alternating paths, as in the proof of Lemma 4.3.

(3) The node-joining operation preserves the strong connectedness of the components as one checks from Definition 5.7(d). By repeating this operation, one obtains a directed graph-labelled tree that defines $G$ and consists of one "central" strongly connected component and leaves. This component is isomorphic to $G$, hence $G$ is strongly connected.

Proposition 5.10 : Let $G$ be defined by a directed graph-labelled tree $\mathcal{T}$. Then $G$ is strongly connected if and only if all components of $\mathcal{T}$ are strongly connected.

Proof : The "if" direction is proved in the previous proposition. For the converse, let $x$ and $y$ be distinct vertices of a component $H_{u}$. Let $s \in A^{+}(x) \cap L_{T}$ and $t \in A^{-}(y) \cap L_{T}$. There is a path in $G$ from $s$ to $t$. Each arc of this path corresponds to an alternating path in $S(\mathcal{T})$. The concatenation of these paths is an alternating walk in $S(\mathcal{T})$. It is not necessarly a path because some edges of the tree may be traversed twice. (In the example of Figure 6 the path $2 \longrightarrow$ $6 \longrightarrow 7 \longrightarrow 4$ corresponds to a walk in $S(\mathcal{T})$ (Figure 5 ) that traverses twice the edge between $H_{u}$ and $H_{w}$ ). This walk must enter $H_{u}$ first via $x$ and exit it last via $y$. Its arcs belonging to $H_{u}$ form a directed path from $x$ to $y$. Hence, $H_{u}$ is strongly connected.

Remark 5.11 : Even if $G(\mathcal{T})$ is strongly connected, some components of $\mathcal{T}$ may not be isomorphic to induced subgraphs of $G(\mathcal{T})$ (by contrast with Lemma 4.3(2)). Consider for an example a directed graph-labelled tree having two internal components isomorphic to the directed cycles $\vec{C}_{3}$. It defines $\vec{C}_{4}$ that does not contain $\vec{C}_{3}$ as an induced subgaph. We will compare below the clique-widths of a graph and its components.

\subsection{Evaluating graph-labelled trees by means of substitu- tions.}

We will use the set of labels $D:=\{\perp,+,-, \top\}$.

Definitions 5.12 : Rooted graph-labelled trees and related notions.

(a) Let $\mathcal{T}$ be a directed graph-labelled tree with underlying tree $T$ and $G:=G(\mathcal{T})$. Let us select a node $r \in N_{T}$ and make it a root for $T$. As in

\footnotetext{
${ }^{39}$ The sets $A^{-}(x) \cap L_{T}$ and $A^{+}(x) \cap L_{T}$ may be different. In the example of Figure 7 , we have $A^{-}(a) \cap L_{T}=\{1\}$ and $A^{+}(x) \cap L_{T}=\{0,1\}$.
} 
Definition 4.9(a,b), for $u$ in $N_{T}$, we define $N_{u}:=\left\{x \in N_{T} \mid x \leq_{T} u\right\}$ and $V_{u}:=\left\{x \in L_{T} \mid x \leq_{T} u\right\} \subseteq V_{G}$. Hence, $V_{r}=L_{T}=V_{G}$ and $V_{u}=\{u\}$ if $u \in L_{T}-\{r\}$.

(b) Let $u \in N_{T}$. The leader of a component $H_{u}$ such that $u \neq r$ is the vertex $\rho_{u}(w u)$ denoted by $\bar{u}$, where $w$ is the father of $u$. We define a $D$-graph $H_{u} \backslash \backslash \bar{u}$ as the follows :

if $u \neq r$ and $u$ not leaf, we define $H_{u} \backslash \backslash \bar{u}:=H_{u}-\bar{u}$ where a vertex $x$ is labelled as follows : its label is $\top$ if $x \bar{u}$ and $\bar{u} x$ are in $E_{H_{u}}$, it is + if $x \bar{u} \in E_{H_{u}}$ and $\bar{u} x \notin E_{H_{u}}$, it is - if $\bar{u} x \in E_{H_{u}}$ and $x \bar{u} \notin E_{H_{u}}$, and it is $\perp$ if $x \bar{u}$ and $\bar{u} x$ are not in $E_{H_{u}}$;

if $u \neq r$ and $u$ is a leaf, then $H_{u} \backslash \backslash \bar{u}$ is undefined (or is empty).

if $u=r$, we define $H_{u} \backslash \backslash \bar{u}:=H_{r}$, and all its vertices as dead (we use the notation $H_{u} \backslash \backslash \bar{u}$ for uniformity, although $\bar{u}$ is not defined).

As in Definition 4.9, the graphs $H_{u} \backslash \backslash \bar{u}$ depend on the chosen root $r$.

(c) If $u \in N_{T}$, we define $G_{u}:=G\left[V_{u}\right]$ labelled as follows:

If $u=r$, all vertices of $G_{u}=G$ are dead.

Otherwise, a vertex $x$ has label $\top$ if there are alternating paths from $x$ to $\bar{u}$ and from $\bar{u}$ to $x$; it has label + if there is an alternating path from $x$ to $\bar{u}$ and no such path from $\bar{u}$ to $x$; it has label - if there is an alternating path from $\bar{u}$ to $x$ and no such path from $x$ to $\bar{u}$ and label $\perp$ if there are no alternating paths between $x$ and $\bar{u}$.

The following lemma, stated for the objects of the previous definition, generalizes Lemma 4.10.

Lemma 5.13: If $u \in N_{T}-L_{T}$ has sons $u_{1}, \ldots, u_{p}$ and the corresponding $p$ vertices of $H_{u} \backslash \backslash \bar{u}$ are $x_{1}, \ldots, x_{p}$ (that is, $\left.x_{i}:=\rho_{u}\left(u u_{i}\right)\right)$, then we have $G_{u}=$ $\left(H_{u} \backslash \backslash \bar{u}\right)\left[x_{1} \leftarrow G_{u_{1}}, \ldots, x_{p} \leftarrow G_{u_{p}}\right]$.

Proof : Let $K:=\left(H_{u} \backslash \backslash \bar{u}\right)\left[x_{1} \leftarrow G_{u_{1}}, \ldots, x_{p} \leftarrow G_{u_{p}}\right]$. As in the proof of Lemma 4.10, the vertex sets of $G_{u}$ and $K$ are the same and the arcs of $G_{u_{i}}$ are the same as in $G_{u}$ and $K$.

We consider $x$ in $G_{u_{i}}$ and $y$ in $G_{u_{j}}, j \neq i$. If $x y$ is an $\operatorname{arc}$ of $G$, there is an alternating path from $x$ to $y$. It must go through $H_{u}$ via the $\operatorname{arc} \rho_{u}\left(u_{i} u\right) \rho_{u}\left(u_{j} u\right)=$ $x_{i} x_{j}$ in $H_{u} \backslash \backslash \bar{u}$. This path goes through the leader $\rho_{u_{i}}\left(u_{i} u\right)$ of $H_{u_{i}}$. Hence, $x$ is alive in $G_{u_{i}}$ and has label + or $\top$. Similarly, $y$ has label - or $\top$ in $G_{u_{j}}$. Hence $x y$ is an arc of $K$.

Conversely, if $x y \in E_{K}$, then $x_{i} x_{j}$ is an arc of $H_{u} \backslash \backslash \bar{u}, x$ has label + or $\top$ in $G_{u_{i}}$ and $y$ has label - or $\top$ in $G_{u_{j}}$. Going back to definitions, we have an 
alternating path from $x$ and $y$ built from alternating paths from $x$ to $x_{i}$, and from $x_{j}$ to $y$, and the $\operatorname{arc} x_{i} x_{j}$. Hence, $x y$ is an $\operatorname{arc}$ of $G$, hence of $G_{u}$.

It remains to compare the vertex labels in $K$ and in $G_{u}$.

Let $x$ be a vertex of $G_{u_{i}}$ labelled by + in $G_{u}$. There is an alternating path from $x$ to the leader $\bar{u}$ of $H_{u}$ and no such path from $\bar{u}$ to $x$. Hence, there is an alternating path from $x$ to $\overline{u_{i}}$ and an arc in $H_{u}$ from $\rho_{u}\left(u u_{i}\right)$ to $\bar{u}$. Hence the label of $\rho_{u}\left(u u_{i}\right)$ is either + or $\top$. The label of $x$ in $G_{u_{i}}$ is either either + or $\top$, hence its label in $K$ is either + or $\top$. If it would be $T$, we would have an arc in $H_{u}$ from $\bar{u}$ to $\rho_{u}\left(u u_{i}\right)$ and an alternating path from $\bar{u}$ to $x$ and $x$ would have label $\mathrm{T}$ in $G_{u}$. Hence $x$ has label + in $K$.

The proofs are similar for the other labels.

Theorem 5.14 : Let $G$ be defined by directed graph-labelled tree $\mathcal{T}$ whose components have clique-width at most $k$. Then $\operatorname{cwd}(G) \leq 8 k+1$.

Proof: From Proposition 5.6 and Lemma 5.13, along the lines of Theorem 4.11.

If one chooses a leaf as root $r$, and its unique son is $u$, then $G=\Lambda^{\prime}\left(\perp, G_{u}\right)$ where $\Lambda^{\prime}(G, H):=\sigma\left[K, x_{1}, x_{2}\right]$ and $K$ consists of the the arcs $x_{1} x_{2}$ and $x_{2} x_{1}$, with $x_{1}$ is labelled by $\top$ and $x_{2}$ by $\perp$. Hence, $\Lambda^{\prime}$ generalizes for $\{\top, \perp,+,-\}$ graphs the operation $\Lambda$ of Definition 2.5.

Example 5.15 : There exist graph-labelled trees $\mathcal{T}$ that have components of arbitrary large clique-width but define graphs without arcs, hence of cliquewidth 1 . For an example, we take $\mathcal{T}$ to be a non-leaf-rooted graph-labelled tree with any connected graph $H$ as root component. We attach to each vertex $x$ of $H$ a path of the form $x-v_{x} \longrightarrow v_{x}^{\prime}-w_{x}^{\prime} \longleftarrow w_{x}-\widetilde{x}$ (cf. Example 5.8(2)). Then $G(\mathcal{T})$ consists of the isolated vertices $\widetilde{x}$ because there is no directed alternating path between $\widetilde{x}$ and any different $\widetilde{y}$.

For strongly connected graphs, we have a better situation.

Proposition 5.16 : There is a function $f$ such that $c w d(H) \leq f(c w d(G))$ whenever $G$ is strongly connected and $H$ is a component of some directed graphlabelled tree that defines it.

We need some technical definitions.

Definition 5.17 : Arc contractions.

Contracting the edges or arcs of set $F$ in a graph $G$ yields a graph $G \backslash F$, usually defined up to isomorphism or with vertices that are subsets of $V_{G}$. This is not convenient for our proof that uses logic.

If $F$ is a set of arcs of a directed graph $G$, we denote by $x \sim_{F} y$ the fact that vertices $x$ and $y$ are linked by a path whose arcs can be traversed in either direction. If $X$ is a set of vertices containing one and only one vertex of each equivalence class of $\sim_{F}$, we denote by $G \backslash(F, X)$ the directed graph $H$ such that 


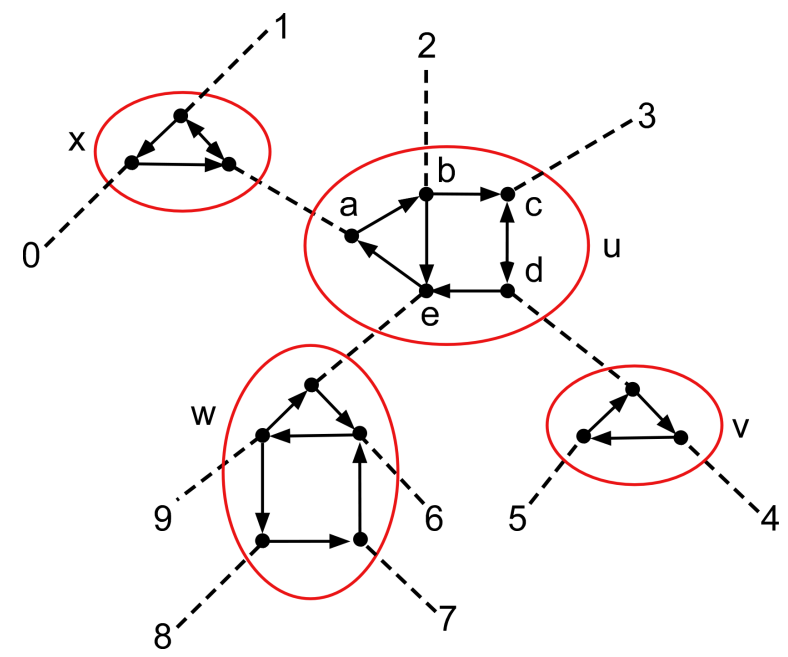

Figure 7: The directed graph-labelled tree $\mathcal{T}$ of Example 5.19.

$V_{H}:=X$ and $x y \in E_{H}$ if and only if $x \neq y$ and there is in $G$ an arc $\overline{x y}$ such that $\bar{x} \sim_{F} x$ and $\bar{y} \sim_{F} y$.

Proof of Proposition 5.16 : Let $G$ be strongly connected and defined by a directed graph-labelled tree $\mathcal{T}$. Let $H_{u}$ be a component not reduced to a single vertex. We will construct an induced subgraph $G^{\prime}$ of $G$ such that $H_{u}$ is isomorphic to $G^{\prime} \backslash F$ for some set of arcs $F$. That $G^{\prime} \backslash F$ is in some sense monadic second-order definable from $G$ yields the existence of $f$ by Corollary $7.38(2)$ of [14].

Step 1 : By performing node-joinings (cf. Definition 5.7(d)) that do not involve $H_{u}$, we obtain a directed graph-labelled tree $\mathcal{T}^{\prime}$ that defines $G$, such that $u \in N_{T^{\prime}}, H_{u}^{\prime}=H_{u}$ and all nodes that are not leaves are neighbours of $u$. The graph-labelled tree of Figure 7 is of this type.

Step 2 : From now on, we assume that $\mathcal{T}$ satisfies this condition. We recall from Definition 5.7 that we identify $v$ and the vertex in $H_{v}$ if $H_{v}$ has only one vertex, so that $L_{T}=V_{G}$. We will define an injective mapping $h: V_{H_{u}} \rightarrow V_{G}$, an induced subgraph $G^{\prime}$ of $G$ whose vertex set contains $h\left(V_{H_{u}}\right)$ and a set $F \subseteq E_{G^{\prime}}$ such that $h$ is an isomorphism $H_{u} \rightarrow G^{\prime} \backslash(F, X)$ for some set $X$.

For each $x \in V_{H_{u}}$, we define $h(x)$ and a few related objects by one of the following three cases.

Case 1: $x=\rho_{u}(u y)$ where $u y \in E_{T}$ and $y \in L_{T}=V_{G}$. Then, we define $h(x):=y$. In Figure 7 , we have $h(b)=2$ and $h(c)=3$ by these conditions. 


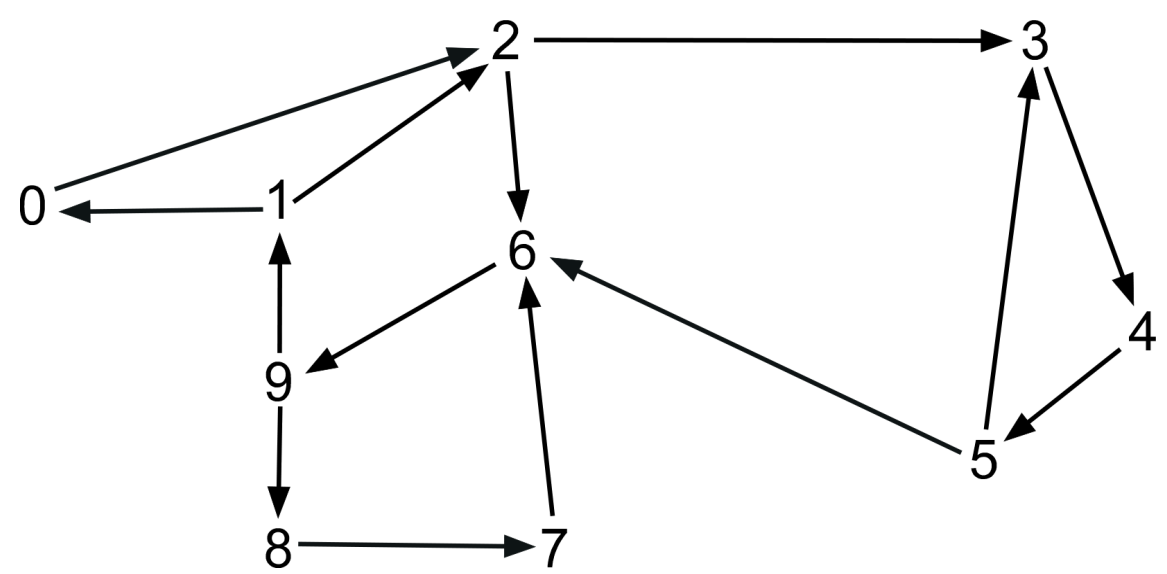

Figure 8: The strongly connected graph $G$ defined by the graph-labelled tree of Figure 7 .

Case 2 : $x=\rho_{u}(u v)$ where $v \notin L_{T}$ and there are in $H_{v}$ two opposite arcs between $z:=\rho_{v}(u v)$ and some vertex $\bar{z}$. We choose one such $\bar{z}$ and we define $h(x)$ as the vertex $y \in V_{G}=L_{T}$ such that $\rho_{v}(v y)=\bar{z}$. In Figure 7 , we have in this way $h(a)=1$.

We denote by $Y_{0}$ the set of vertices $h(x)$ obtained by these two cases.

Case 3: $x=\rho_{u}(u v)$ where $v \notin L_{T}$ and the conditions of Case 2 do not hold. We let $z:=\rho_{v}(u v)$. There is in $H_{v}$ a directed cycle $z \rightarrow z_{1} \rightarrow \ldots \rightarrow z_{p} \rightarrow z$ with $p \geq 2$ because this graph is strongly connected by Proposition 5.10 . We choose one such that $p$ is minimal. It follows that there is no arc in $H_{v}$ between $z$ and any of $z_{2}, \ldots, z_{p-1}$, and from $z_{i}$ to $z_{j}$ if $j>i+1$.

Let $y_{1}, \ldots, y_{p}$ be the vertices of $G$ such that $z_{i}=\rho_{v}\left(v y_{i}\right)$ for each $i$. We have a path $y_{1} \rightarrow \ldots \rightarrow y_{p}$ such that there is no arc $y_{i} y_{j}$ with $j>i+1$. We define $h(x):=y_{1}, h^{\prime}(x):=y_{p}, V_{x}:=\left\{y_{2}, \ldots, y_{p-1}\right\}$ and $F_{x}$ as the set of arcs from $\{h(x)\} \uplus V_{x}$ to $\left\{h^{\prime}(x)\right\} \uplus V_{x}$.

In Figure 7, we have in $H_{w}$ a minimal directed cycle $\rho_{w}(u w) \rightarrow z_{1} \rightarrow z_{2} \rightarrow$ $\rho_{w}(u w), y_{1}=h(e)=6$ and $y_{2}=h^{\prime}(e)=9$.

We denote by $Y_{1}$ and $Y_{2}$ the sets of such vertices $h(x)$ and, respectively, $h^{\prime}(x)$. We denote by $Y_{3}$ the union of the sets $V_{x}$, and by $F$ the union of the sets $F_{x}$. We define $G^{\prime}$ as the induced subgraph $G\left[Y_{0} \uplus Y_{1} \uplus Y_{2} \uplus Y_{3}\right]$ and $X:=Y_{0} \uplus Y_{1}$.

In Figure 7, we have $Y_{0}=\{1,2,3\}, Y_{1}=\{4,6\}, Y_{2}=\{5,9\}$ and $Y_{3}=\emptyset$. The corresponding graph is in Figure 8. The set $F$ consists of the arcs 45 and 69. The vertex set of $G^{\prime}$ is $\{1,2,3,4,5,6,9\}$. By contracting the arcs 45 and 69 of $G^{\prime}$, we obtain a graph isomorphic to $H_{u}$ by : $a \longmapsto 1, b \longmapsto 2, c \longmapsto 3$, $d \longmapsto 4, e \longmapsto 6$.

Claim 1 : The mapping $h$ is an isomorphism : $H_{u} \rightarrow G^{\prime} \backslash(F, X)$. 
Proof : By the definitions, $h$ is a bijection : $V_{H_{u}} \rightarrow X=V_{G^{\prime} \backslash(F, X)}$.

Let $x x^{\prime}$ be an arc of $H_{u}$. There is in $\mathcal{T}$ an alternating path from $h(x)$ or $h^{\prime}(x)$ to $h\left(x^{\prime}\right)$. For proving this, we should conider the nine cases depending on the three possible cases for defining $h(x)$ and $h\left(x^{\prime}\right)$. We only consider the following two, the others being similar or straightforward.

Case (a): $h(x)$ is defined by Case 2 and $h\left(x^{\prime}\right)$ by Case 3 .

Let $x, z, \bar{z}$ and $y=h(x)$ be as in Case 2. Let $z^{\prime} \rightarrow z_{1}^{\prime} \rightarrow \ldots \rightarrow z_{p^{\prime}}^{\prime} \rightarrow z^{\prime}$, $y_{1}^{\prime}, \ldots, y_{p^{\prime}}^{\prime}$ be as in Case 3 for $x^{\prime}$, with $h\left(x^{\prime}\right):=y_{1}^{\prime}$. There is an alternating path $y-\bar{z} \rightarrow z-x \rightarrow x^{\prime}-z^{\prime} \rightarrow z_{1}^{\prime}-y_{1}^{\prime}$ and $y_{1}^{\prime}=h\left(x^{\prime}\right)$. Hence we have $y y_{1}^{\prime}=h(x) h\left(x^{\prime}\right) \in E_{G^{\prime}}$. This arc is also in $G^{\prime} \backslash(F, X)$.

Case (b) : $h(x)$ and $h\left(x^{\prime}\right)$ are defined by Case 3 . In $H_{v}$ we have a minimal directed cycle $z \rightarrow z_{1} \rightarrow \ldots \rightarrow z_{p} \rightarrow z$ with $p \geq 2$. We let $y_{1}, \ldots, y_{p}$ be the corresponding vertices of $G^{\prime}$ so that we have $h(x):=y_{1}$ and $h^{\prime}(x):=y_{p}$. There is an alternating path $y_{p}-z_{p} \rightarrow z-x \rightarrow x^{\prime}-z^{\prime} \rightarrow y_{1}^{\prime}$. Hence we have $y_{p} y_{1}^{\prime}=$ $h^{\prime}(x) h\left(x^{\prime}\right) \in E_{G^{\prime}}$. As $h^{\prime}(x) \sim_{F} h(x)$, the $\operatorname{arc} h(x) h\left(x^{\prime}\right)$ is in $G^{\prime} \backslash(F, X)$.

Conversely, let $y u$ be an arc of $G^{\prime} \backslash(F, X)$. We have $\overline{y u} \in E_{G^{\prime}}$ such that $\bar{y} \sim_{F} y, \bar{u} \sim_{F} u$ and there is an alternating path from $\bar{y}$ to $\bar{u}$. As $y \neq u, \bar{y}$ and $\bar{u}$ are not in a same set $\left\{h(x), h^{\prime}(x)\right\} \uplus V_{x}$, and so, we have $\bar{y}=h(x)$ or $\bar{y}=h^{\prime}(x)$ and $\bar{u}=u=h\left(x^{\prime}\right)$ for some arc $x x^{\prime}$ of $H_{u}$. It follows that $y=h(x)$, hence $y u=h(x) h\left(x^{\prime}\right)$, which proves that $h$ is an isomorphism.

Step 3: We now prove that the transformation of $G$ into $G^{\prime} \backslash(F, X)$ is monadic second-order definable. We let $G$ and the sets $Y_{0}, Y_{1}, Y_{2}, Y_{3}$ and $F$ be as above in Step 2.

Claim 2 : Let $x$ be as in Case 3. Let $P$ be a directed path in $G^{\prime}$ of the form $y_{1}=h(x) \rightarrow s_{1} \rightarrow s_{2} \rightarrow \ldots \rightarrow s_{q}$ with $s_{1}, \ldots, s_{q-1}$ in $Y_{3}$ and $s_{q} \in Y_{2}$. Then $V_{x}=\left\{s_{1}, \ldots, s_{q-1}\right\}$ and $s_{q}=h^{\prime}(x)$.

Proof. We use the notation of Case 3.

The arc $y_{1} s_{1}$ is defined by an alternating path of the form : $y_{1}-z_{1} \rightarrow$ $t_{1}-t_{1}^{\prime} \rightarrow \ldots-s_{1}$. By the conditions of Case $3, t_{1} \neq z$. Hence $t_{1}^{\prime}=s_{1}$. We may have $t_{1}=z_{p}$. In this case $s_{1}=y_{p}=h^{\prime}(x), V_{x}=\emptyset$ and the assertion holds. Otherwise, $t_{1}$ is some $z_{i}$. We cannot have $i>2$ by the minimality of $p$ (cf. Case $3)$. Hence $t_{1}=z_{2}$ and $s_{1}=y_{2}$.

We now consider $y_{2} s_{2}$, the next arc on $P$. We have an alternating path $y_{2}-z_{2} \rightarrow t_{2}-t_{2}^{\prime} \rightarrow \ldots-s_{2}$. By the definitions, if $t_{2}=z_{p}$, then $s_{2}=y_{p}=h^{\prime}(x)$, $V_{x}=\left\{y_{2}\right\}$ and the assertion holds. Otherwise, $t_{2}$ is some $z_{i}$. We cannot have $i=1$ or 2 , otherwise $P$ is a cycle, and neither $i>3$ by the minimality of $p$. Hence $t_{2}=z_{3}$ and $s_{2}=y_{3}$. The proof continues similarly. Considering the next arc $y_{3} s_{3}$ yields that $s_{3}=y_{4}$, either in $Y_{2}$ (in that case $y_{4}=y_{p}=h^{\prime}(x)$ ) or in $Y_{3}$.

Finally, we can prove that the directed path $y_{1}=h(x) \rightarrow s_{1} \rightarrow s_{2} \rightarrow \ldots \rightarrow s_{q}$ is identical to $y_{1} \rightarrow y_{2} \rightarrow y_{3} \rightarrow \ldots \rightarrow y_{p}=h^{\prime}(x)$. We have $V_{x}=\left\{s_{1}, \ldots, s_{q-1}\right\}=$ $\left\{y_{2}, y_{3}, \ldots, y_{p-1}\right\}$ and $s_{q}=y_{p}=h^{\prime}(x)$.

The graph $G^{\prime} \backslash(F, X)$ is obtained from $G$ by fusing the vertices $h(x)$ and $h^{\prime}(x)$ for all vertices $x$ as in Case 3, by keeping $h(x)$ as result of such a fusion 
and removing all vertices not in $X:=Y_{0} \cup Y_{1}$. Our next aim is to describe the pairs $\left(h(x), h^{\prime}(x)\right)$ by a monadic second-order formula.

Claim 3 : There exists a monadic second-order formula $\theta\left(Y_{0}, Y_{1}, Y_{2}, Y_{3}, y, z\right)$ such that, if $G, Y_{0}, Y_{1}, Y_{2}, Y_{3}$ are as in Step 2, then for every two vertices $y, z$ of $G$, we have :

$$
\begin{aligned}
& y=h(x) \text { and } z=h^{\prime}(x) \text { for some vertex } x \text { of } H_{u} \text { if and only if } \\
& G \models \theta\left(Y_{0}, Y_{1}, Y_{2}, Y_{3}, y, z\right) .
\end{aligned}
$$

Proof : By Claim 2, the formula $\theta\left(Y_{0}, Y_{1}, Y_{2}, Y_{3}, y, z\right)$ need only express the following :

$y \in Y_{1}, z \in Y_{2}$ and there exists a directed path from $y$ to $z$ whose intermediate vertices are in $Y_{3}$.

Since transitive closure, whence path properties, can be expressed in monadic second-order logic (cf. [14]), we can construct such a formula $\theta$.

To finish the proof, we define $\bar{\theta}\left(Y_{0}, Y_{1}, Y_{2}, Y_{3}, y, z\right)$ as :

$$
y=z \vee \theta\left(Y_{0}, Y_{1}, Y_{2}, Y_{3}, y, z\right) \vee \theta\left(Y_{0}, Y_{1}, Y_{2}, Y_{3}, z, y\right) \text {. }
$$

and $\alpha\left(Y_{0}, Y_{1}, Y_{2}, Y_{3}, y, z\right)$ as :

$$
\exists \bar{y}, \bar{z} \cdot\left[\bar{\theta}\left(Y_{0}, Y_{1}, Y_{2}, Y_{3}, \bar{y}, y\right) \wedge \bar{\theta}\left(Y_{0}, Y_{1}, Y_{2}, Y_{3}, \bar{z}, z\right) \wedge e d g(y, z)\right] .
$$

Given a directed graph $G$ and pairwise disjoint sets of vertices $Y_{0}, Y_{1}, Y_{2}, Y_{3}$, we define $H\left(Y_{0}, Y_{1}, Y_{2}, Y_{3}\right)$ as the directed graph with vertex set $Y_{0} \cup Y_{1}$ and arcs $y z$ such that $G \models \alpha\left(Y_{0}, Y_{1}, Y_{2}, Y_{3}, y, z\right)$. In the words of [14], this graph is obtained from $G$ by a monadic second-order transduction taking as arguments (called there parameters) pairwise disjoint sets of vertices $Y_{0}, Y_{1}, Y_{2}, Y_{3}$. By Corollary 7.38(2) of [14], there exists a function $f$ such that $\operatorname{cwd}\left(H\left(Y_{0}, Y_{1}\right.\right.$, $\left.\left.Y_{2}, Y_{3}\right)\right) \leq f(\operatorname{cwd}(G))$ for all such graph $G$ and sets $Y_{0}, Y_{1}, Y_{2}$ and $Y_{3}$. If $G, H_{u}, Y_{0}, Y_{1}, Y_{2}$ and $Y_{3}$ are $^{40}$ as in Steps 1 and 2, then $H\left(Y_{0}, Y_{1}, Y_{2}, Y_{3}\right)$ is isomorphic to $H_{u}$. This completes the proof.

This proof does not give a good bound for $f$. It is an open question whether $\operatorname{cwd}\left(H_{u}\right) \leq \operatorname{cwd}(G)$ or even $\operatorname{cwd}\left(H_{u}\right)=O(\operatorname{cwd}(G))$ in such a case.

Remark 5.18 : The special case of Proposition 5.16 where $H_{u}$ is a component of the canonical split-decomposition of a strongly connected graph can be derived from Theorem 4.21 in [7]. The proof of Proposition 4.16 of [7] giving that result is incorrect : Lemma A.2.3 shows correctly that $\operatorname{cwd}(H) \leq 4 \operatorname{cwd}(G)$ if $H$ is obtained from $G$ by fusing any two vertices, but, in order to prove the statement, one must fuse the vertices of several pairs (as we do above for defining $G^{\prime} \backslash(F, X)$ from $\left.G^{\prime}\right)$, hence, one does not obtain a bounding function $f$ as claimed.

\footnotetext{
${ }^{40}$ For some sets $Y_{0}, Y_{1}, Y_{2}, Y_{3}$, the graph $H\left(Y_{0}, Y_{1}, Y_{2}, Y_{3}\right)$ may not be isomorphic to any component $H_{u}$ of a graph-labelled tree. Nevertheless $\operatorname{cwd}\left(H\left(Y_{0}, Y_{1}, Y_{2}, Y_{3}\right)\right) \leq f(\operatorname{cwd}(G))$
} 


\subsection{Related work}

Kanté and Rao have defined in [35] the displit decomposition of a directed graph. For an undirected graph, it is the same as the split decomposition. It is incomparable with the split decomposition of [18] because the prime components are different. However, every connected directed graph has a unique decomposition. Furthermore, for an appropriate notion of rank-width for directed graphs, they obtain that the rank-width of a graph is the least upper-bound of the rankwidths of the components of its displit decomposition (cf. Remark 4.15).

They also characterize the directed graphs of rank-width at most 1 in a way that generalizes the various characterizations of distance-hereditary graphs, in particular that of [37].

We think that the results of this section and those of [7] about the existence of monadic second-order transformations between directed graphs and their canonical split decompositions can be extended to displit decompositions.

\section{Conclusion}

Our purpose was to clarify the relationships between split-decompositions for directed and undirected graphs, substitutions to vertices and the related graph grammars, and also to obtain good bounds on the clique-widths of the defined graphs. For doing that we have generalized, in Definitions 2.1 and 5.1, the notion of substitution used in the theory of modular decomposition .

One open problem is the study of the operations defined in Definition 2.5 and their equational properties.

The methods of Section 5 should help to investigate particular classes of directed graphs regarding their clique-width and their generation by unambigous grammars (if possible), along the lines of Section 4.5. Manipulating grammars so as to reach unambiguity in view of counting and random generation has proved useful in Section 4.5. It would be interesting to investigate in a similar way the undirected graphs whose prime components are cycles. These graphs have bounded clique-width by Theorem 4.14 and the upper-bound of 4 to the clique-width of cycles. This fact encourages to try to define them by grammars.

\section{References}

[1] H.-J. Bandelt and H. Mulder, Distance-hereditary graphs, Journal of Combinatorial Theory, Series B, 41 (1986) 182-208.

[2] A. Brandstädt and V.B. Le, Structure and linear-time recognition of 3-leaf powers. Inf. Process. Lett. 98 (2006) 133-138.

[3] M.S. Chang, S.Y. Hsieh and G.H. Chen, Dynamic programming on distance-hereditary graphs, Proceedings of ISAAC 1997, Algorithms and Computation, Lec. Notes Comput. Sci 1350, Springer, 1997, 344-353. 
[4] C. Chauve, É. Fusy and J. Lumbroso, An exact enumeration of distancehereditary graphs. Proceedings of ANALCO (14th Workshop on Analytic Algorithmics and Combinatorics), Barcelona, January 2017, Proceedings pp. $31-45$

[5] S. Cicerone and G. Di Stefano, On the extension of bipartite to parity graphs. Discrete Applied Mathematics 95 (1999) 181-195.

[6] B. Courcelle, An axiomatic definition of context-free rewriting and its application to NLC graph grammars. Theor. Comput. Sci. 55 (1987) 141-181.

[7] B. Courcelle, The monadic second-order logic of graphs XVI : Canonical graph decompositions. Logical Methods in Computer Science 2 (2006).

[8] B. Courcelle, On the model-checking of monadic second-order formulas with edge set quantifications, Discrete Applied Mathematics 160 (2012) 866-887.

[9] B. Courcelle, Clique-width and edge contraction. Inf. Process. Lett. 114 (2014) 42-44.

[10] B. Courcelle, From tree decompositions to clique-width terms, Discrete Applied Mathematics, 248 (2018) 125-144.

[11] B. Courcelle, On quasi-planar graphs : clique-width and logical description. 2018, Discrete Applied Mathematics, this issue, https://doi.org/10.1016/j.dam.2018.07.022.

[12] B. Courcelle and I. Durand, Automata for the verification of monadic second-order graph properties, J. Applied Logic 10 (2012) 368-409.

[13] B. Courcelle and I. Durand, Computations by fly-automata beyond monadic second-order logic, Theor. Comput. Sci, 619 (2016) 32-67.

[14] B. Courcelle and J. Engelfriet, Graph structure and monadic second-order logic, a language theoretic approach, Volume 138 of Encyclopedia of mathematics and its application, Cambridge University Press, June 2012.

[15] B. Courcelle, P. Heggernes, D. Meister, C. Papadopoulos and U. Rotics, A characterisation of clique-width through nested partitions, Discrete Applied Maths, 187 (2015) 70-81.

[16] B. Courcelle and M. Kanté, Graph operations characterizing rank-width, Discrete Applied Mathematics 157 (2009) 627-640.

[17] B. Courcelle, J. Makowsky and U. Rotics, Linear-time solvable optimization problems on graphs of bounded clique-width, Theory Comput. Syst. 33 (2000) 125-150.

[18] W. Cunningham, Decomposition of directed graphs, SIAM. J. on Algebraic and Discrete Methods, 3 (1981) 214-228. 
[19] R. Diestel, Graph theory, Springer, 2006.

[20] R. Downey and M. Fellows, Fundamentals of parameterized complexity, Springer-Verlag, 2013.

[21] I. Durand, TRAG: Term Rewriting Automata and Graphs, a software developped since 2015, http://dept-info.labri.u-bordeaux.fr/ idurand/trag

[22] I. Durand and M. Raskin, TRAG-WEB: Term Rewriting Automata and Graphs (online), Web interface under development, 2018, https://trag.labri.fr

[23] M. Fellows, F. Rosamond, U. Rotics and S. Szeider, Clique-width is NPcomplete. SIAM J. Discrete Math. 23 (2009) 909-939.

[24] E. Fischer J. Makowsky and E. Ravve, Counting truth assignments of formulas of bounded tree-width or clique-width. Discrete Applied Mathematics 156 (2008) 511-529.

[25] P. Flajolet, P. Zimmermann and B. Van Cutsem, A calculus for the random generation of labelled combinatorial structures. Theor. Comput. Sci. 132 (1994) 1-35.

[26] F. Fomin, S. Oum, D. Thilikos, Rank-width and tree-width of $H$-minor-free graphs. Eur. J. Comb. 31 (2010) 1617-1628.

[27] E. Gioan and C. Paul, Split decomposition and graph-labelled trees: Characterizations and fully dynamic algorithms for totally decomposable graphs. Discrete Applied Mathematics 160 (2012) 708-733.

[28] E. Gioan, C. Paul, M. Tedder and D. Corneil, Practical and efficient split decomposition via graph-labelled trees. Algorithmica 69(2014): 789-843.

[29] M. Golumbic and U. Rotics, On the clique-width of some perfect graph classes. Int. J. Found. Comput. Sci. 11 (2000) 423-443.

[30] F. Gurski, The behavior of clique-width under graph operations and graph transformations. Theory Comput. Syst. 60 (2017) 346-376.

[31] M. Habib and C. Paul, A survey of the algorithmic aspects of modular decomposition. Computer Science Review 4 (2010) 41-59.

[32] P. Heggernes, D. Meister and C. Papadopoulos, Characterising the linear clique-width of a class of graphs by forbidden induced subgraphs, Discrete Applied Mathematics 160 (2012) 888-90.

[33] P. Hlinený, S. Oum, D. Seese and G. Gottlob, Width parameters beyond tree-width and their applications. Comput. J. 51 (2008) 326-362.

[34] M. Kanté and M. Rao, The rank-width of edge-coloured graphs. Theory Comput. Syst. 52 (2013) 599-644. 
[35] M. Kanté and M. Rao, Directed rank-width and displit decomposition. Proceedings of WG 2009, Lecture Notes in Computer Science 5911 (2010) 214-225.

[36] D. Meister, Clique-width with an inactive label. Discrete Mathematics 337 (2014) 34-64.

[37] S. Oum, Rank-width and vertex-minors, Journal of Combinatorial Theory, Series B, 95 (2005) 79-100.

[38] V. Ravelomanana and L.Thimonier, Asymptotic enumeration of cographs. Electronic Notes in Discrete Mathematics :7 (2001) 58-61. 